\title{
Stable and unstable equilibrium states in a fishery-aquaculture model
}

\author{
Bergland, Harald; Pedersen, Pål Andreas; Wyller, John
}

\section{Published in:}

Natural Resource Modeling

Link to article, DOI:

$10.1111 / \mathrm{nrm} .12200$

Publication date:

2019

Document Version

Peer reviewed version

Link back to DTU Orbit

Citation (APA):

Bergland, H., Pedersen, P. A., \& Wyller, J. (2019). Stable and unstable equilibrium states in a

fishery-aquaculture model. Natural Resource Modeling, 32(2), [e12200]. https://doi.org/10.1111/nrm.12200

\section{General rights}

Copyright and moral rights for the publications made accessible in the public portal are retained by the authors and/or other copyright owners and it is a condition of accessing publications that users recognise and abide by the legal requirements associated with these rights.

- Users may download and print one copy of any publication from the public portal for the purpose of private study or research.

- You may not further distribute the material or use it for any profit-making activity or commercial gain

- You may freely distribute the URL identifying the publication in the public portal

If you believe that this document breaches copyright please contact us providing details, and we will remove access to the work immediately and investigate your claim. 
This is the peer reviewed version of the following article: Bergland, H., Pedersen, P. A. \& Wyller, J. A. (2018). Stable and unstable equilibrium states in a fishery-aquaculture model. Natural Resource Modeling, which has been published in final form at https://doi.org/10.1111/nrm.12200. This article may be used for noncommercial purposes in accordance with Wiley Terms and Conditions for Use of Self-Archived Versions.

Stable and Unstable Equilibrium States in a Fishery Aquaculture Model

Harald Bergland ${ }^{\mathrm{a}}$, Pål Andreas Pedersen ${ }^{\mathrm{b}, *}$, John Wyller ${ }^{\mathrm{c}, \mathrm{d}}$

${ }^{a}$ School of Business and Economics, Campus Harstad, University of Tromsø - The Arctic University of Norway , P.O. Box 1063 N-9480 Harstad

${ }^{b}$ Nord University Business School, P.O. Box 1490, N-8049 Bodø, Norway

${ }^{c}$ Faculty of Science and Technology, Norwegian University of Life Sciences, P.O. Box 5003, N-1432 As, Norway

${ }^{d}$ Department of Applied Mathematics and Computer Science, Technical University of Denmark, DK-2800 Kongens Lyngby, Denmark

*Corresponding author

Email address: pal.a.pedersen@nord.no (Pål Andreas Pedersen) 


\section{Abstract}

We study interactions between fishery and aquaculture using a $3 D$ generalized Lotka - Volterra model, where we assume that the aquaculture production may affect the growth rate in the fish stock and the productivity in harvesting. In addition, input demands from both marine industries may result in effort competition. We identify conditions for the coexistence of a unique equilibrium state inside the first octant of the phase space and equilibrium states on its boundary. Conditions for stability and instability of these states are also given, thus showing the possibility of having bistability. The equilibrium point inside the first octant is stable if the growth impact on fishery from sea farming is below the potential productivity in harvesting. In the complementary case we have an unstable interior equilibrium, and we may then end up in stable equilibrium states on the boundary, where either the fishery or the aquaculture are wiped out.

Keyword

Fishery, aquaculture, steady state, stability analysis.

\section{Recommendations for Resource Managers}

- More empirical and theoretical research is needed to reveal types of interrelations between fisheries and aquaculture, and their importance for long run stability between the sectors.

- When designing policies for the aquaculture industries, managers should in particular be aware of possible long term harmful effects from aquaculture to fisheries. 
- Increased areas for sea farming reduces the relative profitability of the fishery, and if the area increases above a certain level, this could wipe out the fishery.

\section{Introduction}

The global production volume from the aquaculture industry has increased considerably during the last fifty years, and the growth rate in this sector is still high. In the same period the global catch volume in commercial fisheries, by comparison has been almost constant. China represents more than 60 percent of world aquaculture production and has played a major role in this growth together with Vietnam (FAO, 2016; Abate et al., 2016).

Increased volume of farmed salmon has also contributed to the global growth in aquaculture production the recent years (Abate et al., 2016). Although several of the salmon species are available from both wild and farmed sources, almost all commercially available Atlantic salmon is farmed. Most of the cultured salmon comes from Norway, Chile, Scotland and Canada. Among the salmon farming countries, Norway stands for the largest share of the total production (FAO, 2016). Norwegian salmon production has increased significantly the past decade. ${ }^{1}$

As reported in Read and Fernandes (2003), Svåsand et al. (2016), Osmundsen et al. (2017), and the references therein, aquaculture may have significant effects on the aquatic environment and other user interests or

\footnotetext{
${ }^{1}$ From about 0.4 million tons yearly in 2001 to above 1.3 million tons yearly in 2015 .
} 
stakeholders. The environmental challenges connected to the aquaculture industry have led the governments in the salmon farming countries to practise a relatively strong capacity regulation (Hersoug, 2012; NFD, 2015). Still the growth potential in the aquaculture industry stimulates to move seafarming further offshore, such that area - and environmental conflicts between the aquaculture and the fishery sector may increase (EU, 2012; Read and Fernandes, 2003).

Hoagland et al. (2003) exemplify conflicts between commercial fisheries and aquaculture with cases from the western Mediterranean, the west coast of Ireland, Norway, New Zealand and US. Such conflicts are likely to become a bigger problem as the aquaculture industry continues to expand. In several regions along the Norwegian coast the expansion of farmed salmon production has intensified conflicts with both the wild salmon harvest and the recreational fishing and commercial coastal fisheries, e.g cod, saith and shrimp (Hersoug, 2012; Svåsand et al., 2016).

Some ecological interactions between fishery and aquaculture industry have been analysed by using predator - prey models as a starting point. These models focus on the fodder supply interactions and competition in the seafood product market (Hannesson, 2003; Regnier and Schubert, 2016). In Hoagland et al. (2003) and Mikkelsen (2007) externalities from marine farm activity are included in the one - specie Gordon - Schaefer model and impacts on equilibrium states are discussed. Effort and product market competition are incorporated by Jiang (2010), studying the equilibrium state. McCausland et al. (2006) also incorporate labor markets in a more detailed simulation 
model. Internalisation of environmental cost through an integrated production system, using an interesting case study, is discussed in Whitmarsh et al. (2006).

A wide range of interactions may arise between aquaculture and commercial fisheries. One group of interactions consists of competition regarding space and physical occupation of the ocean, feeding resources and human and financial resources, in addition to consumer market competition for seafood. Another group of interactions is external effects of aquaculture on a fishery. Impacts on the ecosystem from aquaculture activity may have both direct and indirect consequences for commercial fisheries. To a large extent such effects are uncertain. Hence there is a need for more knowledge about these consequences (Svåsand et al., 2016).

This serves as a background for the present paper. We combine possible ecological conflicts and effort competition between traditional fisheries and aquaculture, focusing on the dynamics involved. We consider a single species commercial fishery, and the proposed dynamical model is conceptual in the sense that we explore possible interactions existing between this fishery and an aquaculture activity in a particular coastal (ocean) region. The model which can be seen as an adjusted version of the models presented in Hoagland et al. (2003), Mikkelsen (2007), Foley et al. (2012) and Perrings (2016) is of the $3 D$ generalized Lotka - Volterra type. We examine biological and economic conditions for existence and stability of different equilibria. Unlike the above mentioned works, all possible equilibria are identified, also those on the boundary, and we examine the connection between the unstable 
equilibrium and the stable equilibria on the boundary in order to explain the dynamics in the model. Beyond this application, the generalized Lotka - Volterra type systems have been extensively investigated in population dynamics, see for instance Smith (1995), Hofbauer and Sigmund (1998), Drossel et al. (2004) and Martinez et al. (2006).

We consider two possible types of externality from the aquaculture industry. First of all, we assume that the growth rate in the wild fish stock may be affected by the production level of the aquaculture industry. Several possible direct and indirect ecosystem mechanisms motivate this assumption: (i) The fodder supply to aquaculture may come from a resource (e.g.krill), which also is fed on by the commercial species. For explicit modeling of this mechanism using predator - prey models, see Hannesson (2003) and Regnier and Schubert (2016) and the references therein. (ii) Fish farms activity causes a release of nutrients, particles and fish waste. These releases may effect migration, spawning behavior and spawning quality of the wild fish species. As reported in Svåsand et al. (2016), such impacts are uncertain and the evidences are limited. However, these effects cannot be excluded. (iii) Diseases and treatment of diseases in fish farms, may also cause ecosystem disturbances. Undesirable substances from medicine, or possible other form of treatments, may harm the considered fish stock directly, or harm resources which the commercial species feed on. Possible environmental impacts of chemical use in aquaculture are summarized in Burridge et al. (2010). Also regarding this impact, the evidence so far is limited, but it should not be excluded (Svåsand et al., 2016). (iv) Due to e.g. production accidents 
farmed fish can escape from their facilities into natural environments where they may survive. ${ }^{2}$ This release may have impact on wild fish populations through ecological, genetic and technical interactions (Lorenzen et al., 2012; Liu et al., 2014).

Secondly, we have assumed that the areas occupied by farming may influence productivity, harvest costs or market value in wild fish harvesting ${ }^{3}$. The arguments for this assumption are; $(a)$ The fishing vessels may be displaced from good fishing grounds, occupied by fish farms. This may further cause crowding in other areas, resulting in a need for more effort to catch a certain volume. (b) The release of excess fodder and faeces have impacts on behavior and quality of wild commercial species (Dempster et al., 2009; Svåsand et al., 2016). The stock availability and fish quality may influence harvest operations and market value negatively.

In addition to ecological interdependency between fisheries and aquaculture, there may be different types of economic market dependencies. One type of such dependencies can be the competition for limited input factors

\footnotetext{
${ }^{2}$ In Norwegian salmon and sea trout production about 0.5 mill individuals escaped per year, during the period 1993 to 2005. After 2005 this number are more than halved (Steinset, 2017).

${ }^{3}$ According to NFD (2015) there are approximately 950 approved sites for salmon and sea trout production along the Norwegian coast, which occupy about 80 square kilometers in physical surface area, and the present government aims to increase this area. Later years the aquaculture activity has been given areas further offshore. This development may increase the location conflicts.
} 
between these industries (e.g. in labor and/or capital markets). Such market interactions are treated by Jiang (2010) in a steady state equilibrium model. In another work McCausland et al. (2006) present a more detailed bioeconomic model for fisheries and aquaculture, where labor mobility are included. They argue that aquaculture in rural communities appears to have a positive impact on employment. For instance in the west coast of Scotland aquaculture provides an important source of employment (McCausland et al., 2006), which also is the case in Norway. Several coastal communities in the western and northern part of Norway have a substantial employment share in both aquaculture and fisheries. Hence, we consider the possibility that these industries may compete for effort. There are at least two reasons why such labor market competition may occur. Firstly, both aquaculture and fishery production are located in coastal areas where people have marine and maritime competence that could be used either in sea farming or in fishing. It is likely that the possible earnings from these two industries affect the actual choice of workplace. Secondly, it also seems likely that these seafood sectors might be competing to employ highly educated candidates in marine biology, technology and management.

The present paper is organized as follows: In Section 2 we present our modeling framework. This framework captures the interactions between the aquaculture activity and the wild fishery. Section 3 is devoted to the analysis of the model. Here we transform the actual system to non - dimensional form by means of scaling, before proceeding to the study of existence and stability of equilibrium points. We also elaborate on different special aspects of the 
model. In Section 4 we present numerical illustrations of the dynamics, whereas Section 5 contains concluding remarks and an outlook. Appendix A, Appendix B and Appendix C contain the mathematical details underlying the conclusions presented in Section 3.

\section{Model}

We will consider a fish harvesting industry where the Gordon-Schaefer production function reads

$$
H=q X E_{x}
$$

Here $H$ is the harvesting rate, $X$ the wild fish population density (biomass), $E_{x}$ the harvest effort and $q$ the harvest efficiency rate.

We combine the production function (1) with a logistic growth model. This assumption which is often used in analyses of fisheries represents a simple way of describing the saturation of the population due to the limited food resources available. See Clark (2010); Flaaten (2010). Following the same general principle as underlying Lotka - Volterra type of models for interspecific competing species as outlined in for instance Hofbauer and Sigmund (1998), Murray (2002) and de Roos (2014) and incorporating the possible mechanisms (i) - (iv), described in the previous section, a modified logistic growth equation for the wild fish specie can be formulated as

$$
\frac{d X}{d t}=\sigma X\left(1-\frac{X+\alpha S}{K}\right)-H
$$

Here $\sigma$ is the intrinsic logistic growth rate and $K$ the carrying capacity. The term $\alpha S$ in (2) represents a possible decrease in growth of wild fish due to 
the presence of the aquaculture production. We assume that $\alpha \geq 0$ and $S$ is the aquaculture production volume. When $\alpha S=0$, the equation (2) reduces to the standard logistic equation.

Moreover, we assume that the fishing productivity, $q$, might be negatively affected as the ocean area allocated to the aquaculture industry, $a$, increases. This assumption relates the mechanisms (a) and (b) described in Section 1, where we emphasize that the fishing activity may be displaced from steadily more good fishing grounds as the ocean area occupied by sea farming increases. In order to simplify, we assume that these mechanisms can be modeled by means of the linear function

$$
q=q(a)=\rho-\varrho a, \quad \rho>0, \quad \varrho \geq 0, \quad \rho>\varrho a
$$

In most countries the public authorities decide the ocean area that could be disposed to aquaculture production as an important part of the governmental policy in coastal zones. See for instance EU (2012) and Hersoug (2012). This means that the aquaculture industry does not directly affect the area allocated to sea farming production ${ }^{4}$.

The equations (2) and (3) together yield two possible negative externalities from the aquaculture to the fishery. Equation (2) models an externality on the biological growth potential in the wild fish stock, while (3) describes a negative externality from the area usage in aquaculture which may cause

\footnotetext{
${ }^{4}$ For instance, in Norway, the different sea farming firms may apply to the public authorities for new sea locations for farming plants. However, it is a governmental decision whether such a permission is granted or not.
} 
more inefficient fishery operations.

Regarding the aquaculture industry, we assume that the production volume, $S$, is linearly increasing in the effort allocated to sea farming, $E_{s}$ :

$$
S=S\left(a, E_{s}\right)=r a E_{s}
$$

Here $r$ is an exogenous efficiency parameter and $a$ is measuring the ocean area available for sea farming, exogenous for the aquaculture industry. For a given level $a$, the industry may increase its production by inserting more effort. Notice that the expression (4) must be regarded as an aggregated production function for the whole industry. The assumption of exogenous and constant marginal productivity in sea farming is made to obtain an easily tractable model.

Due to the discussions related to regional labor markets in coastal areas in Section 1, we suppose that the sea food industries have to compete in the labor market to recruit employees. The total labor supply in the market, $E$, is assumed to be a linear, increasing function of the wage level, $\omega$. The supply function written in the wage form is thus supposed to be given as

$$
\omega=w+\mu E
$$

where, $w>0$ and $\mu \geq 0$. Additionally, we assume that the supply is equal to the total demand, i.e. $E=E_{x}+E_{s}$. The parameter $\mu$ expresses how much the wage has to increase in order to impose one more unit labor into the market. If the labor supply is perfectly elastic, $\mu$ is equal to zero, and the wage level is given by $w$. Now, by taking the effort market equilibrium 
in (5) into account, we find that the profit $\pi_{s}$ in the aquaculture industry is given as a function of the efforts in both industries:

$$
\pi_{s}\left(E_{x}, E_{s}\right)=p_{s} r a E_{s}-\omega E_{s}=\left(p_{s} r a-w\right) E_{s}-\mu E_{s}^{2}-\mu E_{x} E_{s}
$$

Here $p_{s}$ denotes the market price per unit of production in sea farming.

The profit $\pi_{x}$ in the fish harvest industry can now be written as a function of stock volume and effort in both industries when taking the harvest externality (3) and the effort market mechanism (5) into account:

$$
\pi_{x}\left(X, E_{x}, E_{s}\right)=\left(p_{x}(\rho-\varrho a) X-w\right) E_{x}-\mu E_{x}^{2}-\mu E_{s} E_{x}
$$

Here $p_{x}$ denotes the unit price for wild fish. Many authors have conducted interesting analyses of fishery - aquaculture dynamics where the demands for wild and farmed fish are dependent on prices in both sea food markets. See for instance Anderson (1985), Ye and Beddington (1996) and Regnier and Schubert (2016). Additionally, interesting seafood market dependency has been investigated in Hannesson (1983) and Steinshamn (2017). They introduce demands in the sea food markets that contain both direct and possible indirect (cross) price effects, and hence end up with models with endogenous output prices. In our analysis, however, where we explicit regard the possibility of an interrelation between the sea food sectors though an input market, we will, for the sake of simplicity, consider both $p_{x}$ and $p_{s}$ as exogenous variables. ${ }^{5}$

\footnotetext{
${ }^{5}$ By introducing interdependent demands, the profitability of an industry becomes de-
} 
We are now in position to prescribe the dynamics of the effort variables, $E_{s}$ and $E_{x}$. In order to simplify, we assume that expansions and contractions of effort in both industries correlate with positive and negative profits, respectively. Similar types of effort enter - exit mechanisms, assuming frictions and delays, are often used in fishery studies. See for example Smith (1969); Chakraborty et al. (2012); Ghosh and Kar (2014); Regnier and Schubert (2016). These adjustments include frictions and delays. In an open access regime we consider an enter - exit mechanism, where the levels of labor in the industries expand when the industry profit is positive, and contract when the industry profit is negative. We assume that also the adjustment of effort in the aquaculture industry is followed by the profit motive. As long as there is a positive profit margin, the effort is increased and when the profit margin is negative, the labor usage is reduced, meaning that the equilibrium, analogously to the harvesting industry, is defined by zero profit ${ }^{6}$. We take these properties into account by suggesting the instantaneous change of rate of both $E_{x}$ and $E_{s}$ to be proportional to the industry profit $\pi_{x}$ and $\pi_{s}$, respectively, i.e.

$$
\frac{d E_{x}}{d t}=\lambda_{x} \pi_{x}\left(X, E_{x}, E_{s}\right), \quad \frac{d E_{s}}{d t}=\lambda_{s} \pi_{s}\left(E_{x}, E_{s}\right)
$$

pendent on the market supply of the other industry. This mechanism is similar to those ones we obtain through input market dependence.

${ }^{6}$ Notice that our assumption of constant marginal productivity in sea farming, for given input and output prices, either gives a positive or a negative profit. As the input price varies due to the level of aggregated demand equal to supply, the long term equilibrium is defined by zero profit. 
The positive proportionality constants $\lambda_{x}$ and $\lambda_{s}$ are determined by the 'speed of adjustment' measuring the intensity of reaction between the labor input and perceived profit in the respective industries. These parameters $\left(\lambda_{x}\right.$ and $\left.\lambda_{s}\right)$ may be different between industries, since the 'speed of adjustment' for each industry depends on conditions which may vary, including regulatory policy in both sectors.

Inserting both production functions, (1) and (4), and the efficiency externality (3) into the wild fishery growth equation (2), we get

$$
\frac{d X}{d t}=X\left(\sigma\left(1-\frac{X+\alpha r a E_{s}}{K}\right)-(\rho-\varrho a) E_{x}\right)
$$

By inserting the profit functions (6) and (7) into (8), we end up with the rate equations

$$
\begin{gathered}
\frac{d E_{x}}{d t}=\lambda_{x} E_{x}\left(\left(p_{x}(\rho-\varrho a) X-w\right)-\mu E_{x}-\mu E_{s}\right) \\
\frac{d E_{s}}{d t}=\lambda_{s} E_{s}\left(\left(p_{s} r a-w\right)-\mu E_{s}-\mu E_{x}\right)
\end{gathered}
$$

The variables and the parameters in the $3 D$ model of differential equations (9) - (11) and their respective interpretations are summarized in Table 1. The fundamental dimensions given in Table 1 are $T$ for time (e.g. year, month), $M$ for mass (e.g. tons, $\mathrm{kg}$ ), $A$ for area (e.g. meter ${ }^{2}, \mathrm{~km}^{2}$ ), $E$ for effort (e.g. employee, capital) and $C$ for currency (e.g. Euro, Yuan). Notice also that the dimensions of the quantities listed in Table 1 are power products of the fundamental dimensions.

Notice that the $3 D$ system of differential equations (9) - (11) is of the generalized Lotka - Volterra type. Such types of systems have been exten- 
sively investigated in the population dynamics and game theoretical literature Hofbauer and Sigmund (1998) with respect to existence of attractors, equilibrium points, bistability and attraction basins. The forthcoming sections will indeed reveal some of these properties.

\section{Analysis of the model}

\subsection{Scaling and general properties of the model}

The starting point is that our model (9) - (11) as well as any model of processes in physics, biology, chemistry, engineering, economics etc. on dimensional form fulfills the following obvious requirements: The terms of the equations have the same dimension (dimensional homogeneity) and the form of the equations is invariant under the change of the fundamental dimensions (form invariance). In accordance with Buckinghams $\Pi$ - theorem, this implies that our model is equivalent with a system in 6 dimensionless parameters and 4 dimensionless variables. The latter parameters and variables are power products of the variables and parameters listed in Table 1. See for example Chapter 1 in (Logan, 1987) for a general exposition of dimension analysis of mathematical models. By transforming the model to dimensionless form we detect power products that influence the dynamical evolution described by the model. From a practical point of view is indeed an advantage to reduce the number of parameters from 13 to 6 . Here we will stress that this reduction is a consequence of the homogeneity and form invariance property of our model. 


\begin{tabular}{|c|c|c|}
\hline $\begin{array}{l}\text { Variables/ } \\
\text { parameters }\end{array}$ & $\begin{array}{l}\text { Biological/ } \\
\text { economical interpretation }\end{array}$ & $\begin{array}{l}\text { Measurement } \\
\text { dimensions }\end{array}$ \\
\hline$t$ & Time & $T$ \\
\hline$X$ & Wild fish population density & $M$ \\
\hline$H$ & Harvesting rate of wild fish & $M T^{-1}$ \\
\hline$K$ & Carrying capacity of the wild fish biomass & $M$ \\
\hline$\sigma$ & Intrinsic growth rate for the biomass & $T^{-1}$ \\
\hline$a$ & Area occupied by aquaculture activity(constant) & $A$ \\
\hline$\alpha$ & Aquaculture production effect on biomass growth & $T$ \\
\hline$\rho$ & Fixed efficient coefficient fishery & $E^{-1} T^{-1}$ \\
\hline$\varrho$ & Harvest efficiency area effect & $A^{-1} E^{-1} T^{-1}$ \\
\hline$r$ & Efficiency coefficient aquaculture & $M A^{-1} E^{-1} T^{-1}$ \\
\hline$E_{x}$ & Labor input in fishery & E \\
\hline$E_{s}$ & Labor input in aquaculture & $E$ \\
\hline$p_{x}$ & Product price fishery & $C M^{-1}$ \\
\hline$p_{s}$ & Product price aquaculture & $C M^{-1}$ \\
\hline$w$ & Exogenous wage rate & $C E^{-1} T^{-1}$ \\
\hline$\mu$ & Market wage rate impacts from the two industries & $C E^{-2} T^{-1}$ \\
\hline$\lambda_{x}$ & Speed of adjustment (wild fishery) & $E C^{-1}$ \\
\hline$\lambda_{s}$ & Speed of adjustment(aquaculture industry) & $E C^{-1}$ \\
\hline
\end{tabular}

Table 1: The fishery - aquaculture model (9) - (11). All the parameters and variables are non-negative. The fundamental dimensions are $T$ for time (e.g. year, month), $M$ (e.g. tons, $k g$ ), $A$ for area (e.g. meter ${ }^{2}, \mathrm{~km}^{2}$ ), $E$ for effort (e.g. employee, capital) and currency $C$ (e.g. Euro, Yuan). 
In our situation with a mathematical model given as a set of differential equations we obtain a dimensionless form of the model in the standard way by means of a scaling technique. See Chapter 1 in Logan (1987) for an exposition on scaling techniques for differential equations. We proceed in the following way: Introduce the dimensionless quantities $\tau, \xi, \eta, \theta$ and $\gamma_{i} ; i=1,2, \ldots 6$ defined by

$$
\begin{gathered}
\tau=\sigma t, \quad X(t)=K \xi(\tau), \quad E_{x}(t)=\frac{\sigma}{\rho-\varrho a} \eta(\tau), \quad E_{s}(t)=\frac{K}{\alpha r a} \theta(\tau) \\
\gamma_{1}=\frac{\lambda_{x} w}{\sigma}, \quad \gamma_{2}=\frac{\lambda_{x} p_{x}(\rho-\varrho a) K}{\sigma}, \quad \gamma_{3}=\frac{\lambda_{x} \mu}{\rho-\varrho a} \\
\gamma_{4}=\frac{\lambda_{x} \mu K}{\sigma \alpha r a}, \quad \gamma_{5}=\frac{\lambda_{s}\left(p_{s} r a-w\right)}{\sigma}, \quad \gamma_{6}=\frac{\lambda_{s}}{\lambda_{x}}
\end{gathered}
$$

We then get

$$
\xi^{\prime}=\xi f(\xi, \eta, \theta), \quad \eta^{\prime}=\eta g(\xi, \eta, \theta), \quad \theta^{\prime}=\theta h(\xi, \eta, \theta)
$$

where $f, g$ and $h$ are the linear functions

$$
\begin{gathered}
f(\xi, \eta, \theta)=1-\xi-\eta-\theta \\
g(\xi, \eta, \theta)=-\gamma_{1}+\gamma_{2} \xi-\gamma_{3} \eta-\gamma_{4} \theta \\
h(\xi, \eta, \theta)=\gamma_{5}-\gamma_{3} \gamma_{6} \eta-\gamma_{4} \gamma_{6} \theta
\end{gathered}
$$

from (9) - (11). Here the notation ' means differentiation with respect to T. ${ }^{7}$

\footnotetext{
${ }^{7}$ Notice that the present model that there are infinitely many ways of scaling the model (Logan, 1987). Notice also that the scaling technique also makes it possible to compare the relative strengths of the different terms in the model. This is an issue dealt with in a forthcoming part of the present paper (Subsubsection 3.4.2).
} 


\begin{tabular}{ll}
\hline \hline Parameter definition & Intepretation \\
\hline \hline$\gamma_{1}=w /\left(\frac{\sigma}{\lambda_{x}}\right)$ & The relative exogenous unit cost of effort. \\
\hline$\gamma_{2}=p_{x}(\rho-\varrho a) K /\left(\frac{\sigma}{\lambda_{x}}\right)$ & $\begin{array}{l}\text { The relative potential gross profit } \\
\text { per unit of effort in the fishery }\end{array}$ \\
\hline$\gamma_{3}=\mu /\left(\frac{\rho-\varrho a}{\lambda_{x}}\right)$ & The relative direct cost impact from fishery \\
\hline$\gamma_{4}=\mu \frac{K}{\alpha r a} /\left(\frac{\sigma}{\lambda_{x}}\right)$ & The relative indirect cost impact \\
& due to stock externality from aquaculture \\
\hline$\gamma_{5}=\left(p_{s} r a-w\right) /\left(\frac{\sigma}{\lambda_{s}}\right)$ & The relative net profit per unit effort \\
\hline$\gamma_{6}=\frac{\lambda_{s}}{\lambda_{x}}$ & in aquaculture \\
\hline$\frac{\gamma_{3}}{\gamma_{4}}=\frac{\sigma \alpha r a}{K(\rho-\varrho a)}$ & Relative speed of adjustment \\
\hline
\end{tabular}

Table 2: Nondimensional parameters. All the parameters except $\gamma_{5}$ are non-negative.

The dimensionless input parameters $\gamma_{1}, \gamma_{2}, \gamma_{3}, \gamma_{4}, \gamma_{5}$ and $\gamma_{6}$ play a crucial role in the present model. A notable feature is that they measure the relative strengths of parameters involved in the model versus some reference values. The interpretation of the dimensionless parameters $\gamma_{1}, \gamma_{2}, \gamma_{3}, \gamma_{4}, \gamma_{5}$ and $\gamma_{6}$ is summarized in Table 2, based on the role of the parameters in the model (9) - (11). The definitions and our interpretation of the scaling parameters in Table 2 are given in order to facilitate the interpretation of the results in the forthcoming analyses.

We next describe some fundamental properties of the nondimensional system 
(13) - (16). Introduce the vector field $\mathbf{F}: \mathbb{R}^{\mathbf{3}} \rightarrow \mathbb{R}^{\mathbf{3}}$ as

$$
\mathbf{F}(\mathbf{x})=\mathbf{D}(\mathbf{x})\{\mathbf{b}-\mathbf{A} \mathbf{x}\}
$$

where

$$
\begin{gathered}
\mathbf{x}=\left(\begin{array}{l}
\xi \\
\eta \\
\theta
\end{array}\right), \quad \mathbf{b}=\left(\begin{array}{c}
1 \\
-\gamma_{1} \\
\gamma_{5}
\end{array}\right) \\
\mathbf{D}(\mathbf{x})=\left(\begin{array}{lll}
\xi & 0 & 0 \\
0 & \eta & 0 \\
0 & 0 & \theta
\end{array}\right), \quad \mathbf{A}=\left(\begin{array}{ccc}
1 & 1 & 1 \\
-\gamma_{2} & \gamma_{3} & \gamma_{4} \\
0 & \gamma_{3} \gamma_{6} & \gamma_{4} \gamma_{6}
\end{array}\right)
\end{gathered}
$$

Then the system (13) - (16) can conveniently be rewritten on the compact vector form

$$
\frac{d \mathbf{x}}{d \tau}=\mathbf{F}(\mathbf{x})=\mathbf{D}(\mathbf{x})\{\mathbf{b}-\mathbf{A} \mathbf{x}\}
$$

We finally point out a well - known property which the system (19) shares with other generalized Lotka - Volterra systems, namely the existence of invariant regions: We first notice that the coordinate axes as well as the coordinate planes in the $\mathbf{x}$ - phase space are invariant regions of the system i.e. orbits emanating from initial conditions located on these axes (in these coordinate planes) will remain on these axes (in these planes). Hence, orbits starting in the first octant $\Sigma_{+}$defined by

$$
\Sigma_{+}=\left\{\mathbf{x} \in \mathbb{R}^{3} ; \quad \xi, \eta, \theta>0\right\}
$$

will remain in $\Sigma_{+}$, which means that $\Sigma_{+}$is an invariant region of the system under consideration. 


\subsection{Equilibrium points of the model}

In this subsection we study the existence of equilibrium points of the model $(13)-(16)$ in the set $\Sigma_{+}$and on its boundary $\partial \Sigma_{+}$. We first search for the equilibrium point on $\partial \Sigma_{+}$. They are given as

$$
\begin{gathered}
Q_{0}=(0,0,0), \quad Q_{1}=\left(0,0, \frac{\gamma_{5}}{\gamma_{4} \gamma_{6}}\right), \quad Q_{2}=(1,0,0) \\
Q_{3}=\left(1-\frac{\gamma_{5}}{\gamma_{4} \gamma_{6}}, 0, \frac{\gamma_{5}}{\gamma_{4} \gamma_{6}}\right), \quad Q_{4}=\left(\frac{\gamma_{1}+\gamma_{3}}{\gamma_{2}+\gamma_{3}}, \frac{\gamma_{2}-\gamma_{1}}{\gamma_{2}+\gamma_{3}}, 0\right)
\end{gathered}
$$

$Q_{0}, Q_{1}, Q_{2}, Q_{3}$ and $Q_{4}$ correspond to the equilibrium points

$$
\begin{gathered}
R_{0}=(0,0,0), \quad R_{1}=\left(0,0, \frac{p_{s} r a-w}{\mu}\right), \quad R_{2}=(K, 0,0) \\
R_{3}=\left(K-\frac{\alpha r a\left(p_{s} r a-w\right)}{\mu}, 0, \frac{p_{s} r a-w}{\mu}\right), \quad R_{4}=\left(\frac{K\left(\frac{w}{\sigma}+\frac{\mu}{q}\right)}{\frac{p_{x} q K}{\sigma}+\frac{\mu}{q}}, \frac{\sigma}{q}\left(\frac{p_{x} q K-w}{p_{x} q K+\frac{\mu \sigma}{q}}\right), 0\right)
\end{gathered}
$$

of the original system (9) - (11), respectively. This clarifies the role of the equilibrium points on the boundary $\partial \Sigma_{+}: R_{1}$ models an equilibrium situation with no wild fish population, no harvest effort and a finite aquaculture effort. The equilibrium effort in aquaculture $E_{s, e}$ is determined by the profitability per unit effort when adjusted for the industries own factor price impact in the absence of fishery activity, i.e the exogenous aquaculture industry profitability, $\left(p_{s} r a-w\right)$, relative to the parameter $\mu$, which expresses how much the wage has to increase in order to impose one more unit labor into the market. $R_{2}$ represents the equilibrium state with no efforts for both industries and the unexploited and unharmed fish biomass will of course be equal to the carrying capacity $\left(X_{e}=K\right)$ in this case. $R_{3}$ is an equilibrium state with no fishery effort but with a finite aquaculture activity. Here we 
notice that this biomass equilibrium, compared to $R_{2}$ is reduced by a term capturing the possible negative biomass growth externality from aquaculture $\left(\alpha r a E_{s, e}\right)$. Finally, but not least $R_{4}$, models an equilibrium situation with no aquaculture effort. The equilibrium biomass $X_{e}$ and the equilibrium fishery effort $E_{x, e}$ are determined by price, cost and growth parameters in a similar manner as in the standard Gordon - Schaefer model. For instance it follows that the equilibrium biomass $X_{e}$ decreases when the fish price $p_{x}$ increases. ${ }^{8}$ We have also made use of the fact that

$$
\gamma_{1} \gamma_{6}+\gamma_{5}=\frac{\lambda_{s} p_{s} r a}{\sigma}>0
$$

to show nonexistence of equilibrium points for which $\xi=0$ and $\eta>0, \theta>0$. In order to ensure that these equilibrium points are located on the boundary of $\Sigma_{+}$, we must have $\gamma_{5}>0$ for $Q_{1}, 0<\frac{\gamma_{5}}{\gamma_{4} \gamma_{6}}<1$ for $Q_{3}$ and $\gamma_{2}>\gamma_{1}$ for $Q_{4}$, respectively. Notice that $Q_{3}$ merges together with $Q_{1}$ when $\gamma_{5} / \gamma_{4} \gamma_{6} \rightarrow 1^{-}$ and with $Q_{2}$ when $\gamma_{5} / \gamma_{4} \gamma_{6} \rightarrow 0^{+}$. We will return to the equilibrium points $Q_{0}, Q_{1}, Q_{2}, Q_{3}$ and $Q_{4}$, when dealing with the stability analysis.

Next we search for possible equilibrium states of the system (17) - (19) in the first octant $\Sigma_{+}$of the phase space. If such states exist, they must satisfy the system of linear equations

$$
\mathrm{Ax}=\mathrm{b}
$$

\footnotetext{
${ }^{8}$ If $\mu=0$, the wage level is given by $w$. In this case the equilibrium point $R_{4}$ corresponds to $X_{e}=K \xi_{e}=w / q p_{x}$ and $E_{x, e}=\frac{\sigma}{q}\left(1-w / q p_{x} K\right)$ when restoring to the dimensional parameters. This is the open access equilibrium $\left(X_{\infty}, E_{\infty}\right)$ in the standard Gordon Schaefer model. See for example Flaaten (2018).
} 
The linear system (24) has a unique solution called $\mathbf{x}_{e}$ given by

$$
\mathbf{x}_{e}=\mathbf{A}^{-1} \mathbf{b}
$$

Here it is tacitly assumed that $\operatorname{det}(\mathbf{A}) \neq 0$ so that the matrix $\mathbf{A}$ is invertible. Simple computation reveals that

$$
\operatorname{det}(\mathbf{A})=\gamma_{2} \gamma_{6}\left(\gamma_{4}-\gamma_{3}\right)
$$

Hence $\mathbf{x}_{e}$ given by (25) exists if and only if $\gamma_{3} \neq \gamma_{4}$. The components $\xi_{e}, \eta_{e}$ and $\theta_{e}$ of $\mathbf{x}_{e}$ are given as

$$
\begin{gathered}
\xi_{e}=\frac{\gamma_{1} \gamma_{6}+\gamma_{5}}{\gamma_{2} \gamma_{6}} \\
\eta_{e}=\frac{\gamma_{4}}{\gamma_{3}-\gamma_{4}}\left(\xi_{e}-1+\frac{\gamma_{5}}{\gamma_{4} \gamma_{6}}\right) \\
\theta_{e}=\frac{\gamma_{3}}{\gamma_{3}-\gamma_{4}}\left(1-\xi_{e}-\frac{\gamma_{5}}{\gamma_{3} \gamma_{6}}\right)
\end{gathered}
$$

provided $\gamma_{3} \neq \gamma_{4}$. By appealing to (23) and the fact that $\gamma_{2}, \gamma_{6}>0$, we conclude that $\xi_{e}>0$. Moreover, we readily find that

$$
\xi_{e}=\frac{p_{s} r a}{p_{x}(\rho-\varrho a) K}
$$

when restoring to the original parameters i.e by using the expressions listed in Table 2. This fraction is interpreted as the relative aquaculture industry profitability. The nominator $\left(p_{s} r a\right)$ is the revenue per unit effort in the aquaculture industry whereas the denominator $\left(p_{x}(\rho-\varrho a) K\right)$ is the potential revenue per unit effort in the fishery. Higher relative profit for the aquaculture 
industry leads to allocation of effort to aquaculture from the fishery and thereby causes a higher equilibrium biomass.

We choose the parameters $\gamma_{i}, i=1,2, \ldots, 6$ such that $\mathbf{x}_{e} \in \Sigma_{+}$. We notice that the parameters $\gamma_{3}$ and $\gamma_{4}$ play a crucial role when studying the existence of this equilibrium point. More information about the role of these two parameters can be extracted from the structure of the dynamical system (13) - (16): If the parameter vector $\left(\gamma_{1}, \gamma_{2}, \gamma_{3}, \gamma_{4}, \gamma_{5}, \gamma_{6}\right)$ produces the equilibrium point $\left(\xi_{e}, \eta_{e}, \theta_{e}\right)$, then the parameter vector $\left(\gamma_{1}, \gamma_{2}, \gamma_{4}, \gamma_{3}, \gamma_{5}, \gamma_{6}\right)$ yields the equilibrium point $\left(\xi_{e}, \theta_{e}, \eta_{e}\right)$. This reflection symmetry follows from the role of the parameters $\gamma_{3}$ and $\gamma_{4}$ in (13) - (16). An interchange of the parameters $\gamma_{3}$ and $\gamma_{4}$ produces an interchange of the effort coordinates. By appealing to Table 2, the interpretation of this property goes as follows: The demand for one extra unit effort in both industries induces the same cost pressure impact in the effort market. $\gamma_{3}$ captures how the fishery effort directly influences the fishery profit via this market mechanism, while $\gamma_{4}$ captures how the aquaculture effort indirectly affects the fishery profit via the biomass growth mechanism. At the same time we notice that the aquaculture industry has a constant unit profit in the absence of the labor market impact $(\mu=0)$, while the fishery profit is still influenced by the aquaculture industry via a possible harmed biomass growth. Hence, an interchange of the parameters $\gamma_{3}$ and $\gamma_{4}$ alters these mechanisms in a symmetric way and consequently should result in an interchange of the two industries effort coordinates in the interior equilibrium state. We will exploit this symmetry in the numerical study of the dynamical system (17) - (19) in different parameter regimes. 
The transition case $\gamma_{3}=\gamma_{4}$ (corresponding to $\operatorname{det}(\mathbf{A})=0$ ) must be treated separately. Appendix A is devoted to a detailed analysis of this case. By making use of Table 2 we interpret this transition case in the following way: The aquaculture production impact per unit of effort on the wild fish population growth (measured by means of $\sigma \alpha r a$ ) is exactly equal to the potential productivity for the fishing industry i.e. the potential harvest per unit of effort (measured by means of $q K=(\rho-\varrho a) K)$. From the structure of the system (9) - (11) we notice that only the total effort of both industries are determined in this transition case, and from the parametrisation (A.3) in Appendix A we have a continuum of equilibrium points. The condition (A.3) in terms of the dimensional quantities is

$$
E_{x, e}+E_{s, e}=\frac{\sigma}{q}\left(\frac{p_{x} q K-w}{p_{x} q K+\frac{\mu \sigma}{q}}\right)
$$

where

$$
E_{x, e}=\frac{\sigma}{q} \eta_{e}, \quad E_{s, e}=\frac{\sigma}{q} \theta_{e}, \quad \frac{\sigma}{q}=\frac{K}{\alpha r a}
$$

Interestingly, the total effort in this case is equal to the fishery effort in the equilibrium state $R_{4}$. Notice that our system possesses the equilibrium points $Q_{0}, Q_{1}, Q_{2}, Q_{3}$ and $Q_{4}$ given by (21) even in this case. The properties of these points are detailed in Appendix A.

\subsection{Stability of the equilibrium points}

The detailed stability assessments of the boundary equilibrium points $Q_{0}, Q_{1}, Q_{2}, Q_{3}, Q_{4}$, and the interior equilibrium point $\mathbf{x}_{e}$ are presented in Appendix B. Here, we only summarize the main results regarding the 
stability of the equilibrium points: For the parameter regimes $\gamma_{4} \neq \gamma_{3}$, the system (13) - (16) possesses a unique equilibrium point $\mathbf{x}_{e}$ in the first octant $\Sigma_{+}$in the phase space. This equilibrium point is stable (unstable) provided $\gamma_{4}>\gamma_{3}\left(\gamma_{4}<\gamma_{3}\right)$. In accordance with Appendix B, no local bifurcations of the equilibrium point $\mathbf{x}_{e}$ take place in the regimes $\gamma_{4}>\gamma_{3}$ and $\gamma_{4}<\gamma_{3}$. We have only a change of the stability property of $\mathbf{x}_{e}$ when passing $\gamma_{4}=\gamma_{3}$ in the 6 - dimensional parameter space.

When restoring to the definition (12) of the nondimensional parameters, we find that the equilibrium point $\mathbf{x}_{e} \in \Sigma_{+}$corresponds to an equilibrium point $\mathbf{X}_{e}=\left(X_{e}, E_{x, e}, E_{s, e}\right)$ of the system (9) - (11). The condition $\gamma_{4}>\gamma_{3}$ for stability of the equilibrium point $\mathbf{X}_{e}$ is now translated into the condition

$$
a<a_{*}, \quad a_{*} \equiv \frac{\rho}{\varrho+\frac{\sigma \alpha r}{K}}
$$

by means of Table 2 . This condition implies that there is an upper bound $a_{*}$ on the aquaculture area $a$ in order to ensure the stability of the equilibrium point $\mathbf{X}_{e}$. This bound is determined by six of the parameters in the original model, (9) - (11). A notable feature is that a higher carrying capacity $K$ will increase the bound $a_{*}$. The bound is proportional to the carrying capacity, $K$, in the absence of harvest productivity impact $(\varrho=0)$. Moreover, efficient harvest in the fishery, i.e an increase in $\rho$, also results in an increase in $a_{*}$, whereas an increase in the negative harvest efficiency impact from aquaculture (which means an increase in $\varrho$ ) and an increased negative growth externality (i.e. a higher $\alpha$, will both reduce the area $a_{*}$ ). Notice that the stability condition $\gamma_{4}>\gamma_{3}$ also can be expressed in terms of the fraction 
interpreted in Table 2: $\frac{\gamma_{3}}{\gamma_{4}}=\frac{\sigma \alpha r a}{K(\rho-\varrho a)}<1$. This fraction measures the growth impact from aquaculture relative to the fishery productivity. ${ }^{9}$

The stability properties of the points given by (21) and (27) are examined in Appendix B and the results are summarized in Table 3. A notable feature which can be extracted from Table 3 is the nonexistence of the equilibrium point $Q_{3}$ in the regime producing a stable equilibrium point $Q_{1}$. This behavior is to be expected since $Q_{3}$ is an equilibrium state with no fishery effort but with a finite aquaculture activity causing a negative biomass growth externality, while $Q_{1}$ models an equilibrium situation with no wild fish population, no harvest effort and a profitable aquaculture effort. In the complementary regime, $0<\gamma_{5}<\gamma_{4} \gamma_{6}$, for which $Q_{1}$ is unstable, $Q_{3}$ is stable for $\gamma_{2}$ below the threshold value $\gamma_{2}^{*} \equiv \gamma_{4} \frac{\gamma_{1} \gamma_{6}+\gamma_{5}}{\gamma_{4} \gamma_{6}-\gamma_{5}}$, whereas it will be unstable for $\gamma_{2}$ exceeding this threshold value. This seems also reasonable since the equilibrium point

\footnotetext{
${ }^{9}$ Notice that the reproductivity rate $f \equiv \sigma\left(1-\frac{X+\alpha r a E_{s}}{K}\right)-(\rho-\varrho a) E_{x}$ in the wild fishery growth equation (9) in our modelling framework can be viewed as a linear approximation of a more general reproductivity growth function in the population density $X$ and the effort variables $E_{x}$ and $E_{s}$. Here we follow the line of thought in Drossel et al. (2004) and Martinez et al. (2006) with respect to the modelling of a foodweb. This means that the reproductivity function contains terms describing saturating functional responses. This type of chemostat - like conditions of the resource level will indeed alter the equilibrium and stability analysis presented in this paper. In particular, we expect that it will change the interpretation of the result relating the stability of the interior equilibrium to the carrying capacity $K$ (i.e. the condition (30)). Alternative specification of the model regarding (1), (2) or (4) could also be considered. We do not pursue this problem in the present paper, however.
} 


\begin{tabular}{ll}
\hline \hline $\begin{array}{l}\text { Equilibrium points in } \Sigma_{+}, \\
\text {and on its boundary } \partial \Sigma_{+}\end{array}$ & Stability property \\
\hline \hline$Q_{0}=(0,0,0)$ & Unstable \\
\hline$Q_{1}=\left(0,0, \frac{\gamma_{5}}{\gamma_{4} \gamma_{6}}\right)$ & Stable (unstable) if $\gamma_{5}>\gamma_{4} \gamma_{6}\left(0<\gamma_{5}<\gamma_{4} \gamma_{6}\right)$, \\
& stability analysis inconclusive if $\gamma_{5}=\gamma_{4} \gamma_{6}$ \\
\hline$Q_{2}=(1,0,0)$ & Stable if $\gamma_{2}<\gamma_{1}$ and $\gamma_{5}<0$, stability analysis inconclusive \\
& if $\gamma_{1}=\gamma_{2}$ and $\gamma_{5} \leq 0$, unstable otherwise \\
\hline$Q_{3}=\left(1-\frac{\gamma_{5}}{\gamma_{4} \gamma_{6}}, 0, \frac{\gamma_{5}}{\gamma_{4} \gamma_{6}}\right)$ & Stable if $\gamma_{2}<\gamma_{4} \frac{\gamma_{1} \gamma_{6}+\gamma_{5}}{\gamma_{4} \gamma_{6}-\gamma_{5}}$, stability analysis \\
& inconclusive if $\gamma_{2}=\gamma_{4} \frac{\gamma_{1} \gamma_{6}+\gamma_{5}}{\gamma_{4} \gamma_{6}-\gamma_{5}}$, unstable otherwise. \\
\hline$Q_{4}=\left(\frac{\gamma_{1}+\gamma_{3}}{\gamma_{2}+\gamma_{3}}, \frac{\gamma_{2}-\gamma_{1}}{\gamma_{2}+\gamma_{3}}, 0\right)$ & Stable if $\gamma_{5}<\gamma_{3} \gamma_{6} \frac{\gamma_{1}+\gamma_{3}+\gamma_{3}}{\gamma_{2}}$, stability analysis inconclusive if \\
& $\gamma_{5}=\gamma_{3} \gamma_{6} \frac{\gamma_{1}+\gamma_{3}}{\gamma_{2}+\gamma_{3}}$, unstable otherwise \\
\hline $\mathbf{x}_{e}=\left(\xi_{e}, \eta_{e}, \theta_{e}\right)$ & Stable $($ unstable $)$ if $\gamma_{4}>\gamma_{3}\left(\gamma_{4}<\gamma_{3}\right)$ \\
\hline &
\end{tabular}

Table 3: Equilibrium points of (13) - (16) in $\Sigma_{+}$and its boundary $\partial \Sigma_{+} \cdot \mathbf{x}_{e}$ is given by (27). It is tacitly assumed that $\gamma_{5}>0$ for $Q_{1}, \gamma_{2}>\gamma_{1}$ for $Q_{2}$ and $0<\frac{\gamma_{5}}{\gamma_{4} \gamma_{6}}<1$ for $Q_{3}$, respectively. See Appendix B for a detailed interpretation. 
$Q_{3}$, which represents an equilibrium state with no fishery effort is stable when the potential gross profit in the fishery (measured by means of $\gamma_{2}$ ) is below the threshold value presented in Table 3 , while $Q_{4}$ which is an equilibrium with no aquaculture effort is stable for $\gamma_{5}$ (the net profit in the aquaculture industry) below a certain threshold value. These threshold values depend on the parameters in the model in a complicated way. See Appendix B for a more detailed discussion.

The equilibrium points alternate between being stable and unstable, which means that we can have coexistence of two stable equilibrium points in both these regimes (the bistability issue). Hence the first octant $\Sigma_{+}$of the phase space can be expressed as a union of attraction basins for the compact attractors such as stable equilibrium points. We do not pursue a detailed analysis of the attraction basins in the present paper, but rather conclude that the system under consideration permits a multitude of dynamical scenarios. The reason for this is the parametric complexity of the system: The dynamical evolution depends on the six parameters $\gamma_{1}, \ldots, \gamma_{6}$. These parameters can be varied independently of each other. Notice that these results resemble those ones which have been observed for different generalized Lotka - Volterra systems. See for example Smith (1995) and Hofbauer and Sigmund (1998).

\subsection{Special cases}

To elaborate on the mechanisms present in our model, it is useful to consider simplified versions of the model. In the subsections below we examine how the model functions when we omit the labor market interaction and the 
biomass growth impact, respectively.

\subsubsection{Simplified model with no labor market impact from wild fishery and aquaculture industry}

Let us consider the special case where labor market impact from wild fishery and aquaculture industry is neglected. In the modeling framework (9), (11) and (10) this means that $\mu=0$. This situation corresponds to letting $\gamma_{3}=\gamma_{4}=0$ in the nondimensional setting (13) - (16). The actual model simplifies to

$$
\xi^{\prime}=\xi(1-\xi-\eta-\theta), \quad \eta^{\prime}=\eta\left(-\gamma_{1}+\gamma_{2} \xi\right), \quad \theta^{\prime}=\gamma_{5} \theta
$$

The last equation in this system possesses the solution

$$
\theta(\tau)=\theta_{0} \exp \left[\gamma_{5} \tau\right]
$$

where $\theta_{0}$ denotes the initial condition, $\theta(0)=\theta_{0}$. This means that the model under consideration reduces to the $2 D$ non - autonomous dynamical system

$$
\xi^{\prime}=\xi\left(1-\xi-\eta-\theta_{0} \exp \left[\gamma_{5} \tau\right]\right), \quad \eta^{\prime}=\eta\left(-\gamma_{1}+\gamma_{2} \xi\right)
$$

$\gamma_{5}$, interpreted as the relative net profit per unit of effort in the aquaculture industry, is not sign - definite, it is of interest to study the solution of this system for both negative and positive values of this parameter. In the negative $\gamma_{5}$ - case we have an exponential decay of $\theta$. The solution of the system is in this case expected to approach the solution of the $2 D$ autonomous dynamical system.

$$
\xi^{\prime}=\xi(1-\xi-\eta), \quad \eta^{\prime}=\eta\left(-\gamma_{1}+\gamma_{2} \xi\right)
$$


for large values of $\tau$. We notice that this special case, (34), is actually the standard Gordon - Schaefer model, only extended with a dynamic adjustment mechanism in the effort, i.e a wild fishery without aquaculture, where the fishery profit determines the expansion/contraction of the industry employment.

The system (34) has three equilibrium points $P_{0}=(0,0), P_{1}=(1,0)$ and $P_{2}=\left(\frac{\gamma_{1}}{\gamma_{2}}, 1-\frac{\gamma_{1}}{\gamma_{2}}\right)^{10}$. Table 4 summarizes the stability properties of these equilibrium points as a function of the parameters $\gamma_{1}$ and $\gamma_{2}$. Notice that $P_{2}$ belongs to the fourth quadrant in the $\xi, \eta$ - plane when $\gamma_{1}>\gamma_{2}$. Due to the interpretation in Table 2, where $\gamma_{2}$ is the relative (potential) gross profit per unit of effort in the fishery and $\gamma_{1}$ is the relative unit cost of effort, it is reasonable to assume that $\gamma_{1}<\gamma_{2}$. As well known from the standard Gordon - Schaefer case, the two equilibrium points $P_{1}$ and $P_{2}$ merge together in the transition state $\gamma_{1}=\gamma_{2} . P_{1}$ and $P_{2}$ swap the stability properties as one passes this transition state. The simulations in Section 4 confirm the predictions obtained from the stability analysis of the equilibrium points $P_{0}$, $P_{1}$ and $P_{2}$ : The solutions which approach the stable equilibrium point $P_{2}$ represent damped oscillations.

In the complementary regime $\left(\gamma_{5}>0\right)$, the $\theta$ grows exponentially and unbounded. Finite labor market impact effects $\left(\gamma_{3}, \gamma_{4} \neq 0\right)$ present in the

\footnotetext{
${ }^{10}$ When the labor supply is perfectly elastic, $\mu=0$, and the wage level is given by $w$, then the equilibrium point $P_{2}$ correspond to $\left(w / q p_{x}, \frac{\sigma}{q}\left(1-w / q p_{x} K\right)\right)$ when restoring to the dimensional parameters.
} 


\begin{tabular}{cccc}
\hline \hline Equilibrium points & $P_{0}$ & $P_{1}$ & $P_{2}$ \\
\hline$\gamma_{1}<\gamma_{2}$ & Unstable & Unstable & Stable \\
\hline$\gamma_{1}>\gamma_{2}$ & Unstable & Stable & Unstable \\
\hline \hline
\end{tabular}

Table 4: Stability of the equilibrium points $P_{0}(0,0), P_{1}(1,0)$ and $P_{2}\left(\frac{\gamma_{1}}{\gamma_{2}}, 1-\frac{\gamma_{1}}{\gamma_{2}}\right)$.

complete model (13) - (16) may saturate this growth, however. In the absence of this saturating mechanism, the unbounded growth of $\theta$ will cause increased negative impact in the fish stock growth rate and the productivity in the fish harvesting industry.

3.4.2. Simplified model with low aquaculture production effect on biomass growth.

We want to study the model (9) - (11) in the limit $\alpha \rightarrow 0$. This means that we assume the effect on biomass growth from aquaculture production to be weak (or negligible). Hence, this case isolates the impact from effort market competition between the two industries. We conveniently carry out this study within a nondimensional modeling framework derived by scaling the model (9) - (10). This will enable us to define what it means that the growth impact from aquaculture production is weak (or negligible). The question which naturally arises is if one could use the system (13) - (16) in this study. The choice (12) will not work, as it predicts the amplitude factor in $E_{s}$ to go to infinity as $\alpha \rightarrow 0$. In order to avoid this problem we proceed as follows: We define $\tau, \xi$ and $\eta$ as in (12), but change the amplitude scaling 
of $E_{s}$ to

$$
E_{s}(t)=\frac{\sigma}{\lambda_{x} \mu} \tilde{\theta}(\tau)
$$

The corresponding nondimensional system which we can derive from (9) (10) reads

$$
\tilde{\xi}^{\prime}=\tilde{\xi} F(\tilde{\xi}, \tilde{\eta}, \tilde{\theta}), \quad \tilde{\eta}^{\prime}=\tilde{\eta} G(\tilde{\xi}, \tilde{\eta}, \tilde{\theta}), \quad \tilde{\theta}^{\prime}=\tilde{\theta} H(\tilde{\xi}, \tilde{\eta}, \tilde{\theta})
$$

where $F, G$ and $H$ are the linear functions

$$
\begin{gathered}
F(\tilde{\xi}, \tilde{\eta}, \tilde{\theta})=1-\tilde{\xi}-\tilde{\eta}-\varepsilon \tilde{\theta} \\
G(\tilde{\xi}, \tilde{\eta}, \tilde{\theta})=-\gamma_{1}+\gamma_{2} \tilde{\xi}-\gamma_{3} \tilde{\eta}-\tilde{\theta} \\
H(\tilde{\xi}, \tilde{\eta}, \tilde{\theta})=\gamma_{5}-\gamma_{3} \gamma_{6} \tilde{\eta}-\gamma_{6} \tilde{\theta}
\end{gathered}
$$

Here

$$
\xi=\tilde{\xi}, \quad \eta=\tilde{\eta}, \quad \theta=\varepsilon \tilde{\theta}
$$

and

$$
\varepsilon \equiv \gamma_{4}^{-1}=\frac{\sigma \alpha r a}{K \lambda_{x} \mu}
$$

whereas the remaining parameters are as given in (12). We aim at studying the system $(36)-(37)$ in the regime $0<\varepsilon \ll 1$ for $\tilde{\xi}, \tilde{\eta}, \tilde{\theta} \geq 0$. This regime will be referred to as the regime of weakly aquaculture production effect on the biomass. By using (39), we translate the inequality $0<\varepsilon \ll 1$ into the condition

$$
\alpha \ll \frac{K \lambda_{x} \mu}{\sigma r a}
$$

Table 5 summarizes the results concerning existence and stability of the equilibrium points in the case $\varepsilon=0$. We also show that the phase portrait of the 


\begin{tabular}{ll}
\hline \hline Equilibrium points & Stability property \\
\hline \hline$M_{1}=\left(0,0, \frac{\gamma_{5}}{\gamma_{6}}\right)$ with $\gamma_{5} \geq 0$ & Always unstable. \\
\hline$M_{2}=\left(1,0, \frac{\gamma_{5}}{\gamma_{6}}\right)$ with $\gamma_{5} \geq 0$ & Asymptotically stable if $\gamma_{5}>\max \left\{0, \gamma_{6}\left(\gamma_{2}-\gamma_{1}\right)\right\}$, \\
& stability analysis inconclusive if \\
& $\gamma_{5}=\max \left\{0, \gamma_{6}\left(\gamma_{2}-\gamma_{1}\right)\right\}$, unstable otherwise. \\
\hline$M_{3}=\left(\frac{\gamma_{1}+\gamma_{3}}{\gamma_{2}+\gamma_{3}}, \frac{\gamma_{2}-\gamma_{1}}{\gamma_{2}+\gamma_{3}}, 0\right)$ with $\gamma_{2}>\gamma_{1}$ & Stable if $\gamma_{5}<\gamma_{3} \gamma_{6} \frac{\gamma_{1}+\gamma_{3}}{\gamma_{2}+\gamma_{3}}$, \\
& stability analysis inconclusive if $\gamma_{5}=\gamma_{3} \gamma_{6} \frac{\gamma_{1}+\gamma_{3}}{\gamma_{2}+\gamma_{3}}$, \\
& unstable otherwise. \\
\hline$M_{4}=\left(\tilde{\xi}_{e}, 1-\tilde{\xi}_{e},\left(\frac{\gamma_{5}}{\gamma_{6} \gamma_{3}}-\left(1-\tilde{\xi}_{e}\right)\right) \gamma_{3}\right)$, & \\
$\tilde{\xi}_{e}=\frac{\gamma_{1}}{\gamma_{2}}+\frac{\gamma_{5}}{\gamma_{2} \gamma_{6}}$ & Always asymptotically stable. \\
\hline
\end{tabular}

Table 5: Equilibrium points of (36) - (37) in the $\varepsilon=0$ - limit.

system (36) - (37) in the vicinity of its equilibrium point is mapped one - to - one and onto the phase portrait of the same system (36) - (37) with $\varepsilon=0$ in the vicinity of $M_{4}$. A detailed analysis is presented in Appendix C.

\section{Numerical illustrations}

In this section we illustrate numerically the results of the general analysis presented in the previous sections. We start out by studying the simplified descriptions presented in the previous two subsubsections. We then show different solutions for the full model (13) - (16). Finally, we give an example of public regulation policy as exogenous shift in the area occupied by the aquaculture industry i.e. the parameter $a$. In all the numerical simulations to be presented we let $\gamma_{1}=1$ and $\gamma_{2}=2$. Notice that these values of $\gamma_{1}$ and 


\begin{tabular}{lll}
\hline \hline Parameters & Values & Measurement units \\
\hline \hline$K$ & 2000000 & $M$ \\
\hline$p_{x}$ & 4000 & $C M^{-1}$ \\
\hline$\rho-\varrho a$ & 0.00001 & $E^{-1} T^{-1}$ \\
\hline$\lambda_{x} / \sigma$ & 0.000025 & $E T C^{-1}$ \\
\hline$w$ & 40000 & $C E^{-1} T^{-1}$ \\
\hline
\end{tabular}

Table 6: Reference parameters. The measurement units are $M=$ Tons, $C=$ Euro, $E=$ Employees and $T=Y e a r$.

$\gamma_{2}$ are computed on the basis of the reference values summarized in Table 6 .

\subsection{Simplified versions and characteristics of the model}

First, we illustrate the model (9) - (11) with no labor market impact $\left(\mu=0 \Rightarrow \gamma_{3}=\gamma_{4}=0\right)$. Hence, this case isolates the impact of the biomass growth externality from aquaculture to the wild fishery. The outcome here is crucial depending on the sign of the exogenous relative profit in the aquaculture industry, i.e. on the sign of $\gamma_{5}$. Assuming $\gamma_{5}<0$, i.e. negative relative net profit in aquaculture, we have an exponential decay of $\theta$. Moreover, as pointed out in Subsection 3.4.1 the solution is expected to approach the solution of the Gordon - Schaefer model (34) for large values of $\tau$. The phase portrait of (34) is depicted in Fig.1a and the density evolution in Fig.1b. This simulation confirms the predictions obtained from the stability analysis summarised in Table 4. The solutions approach the stable equilibrium point 


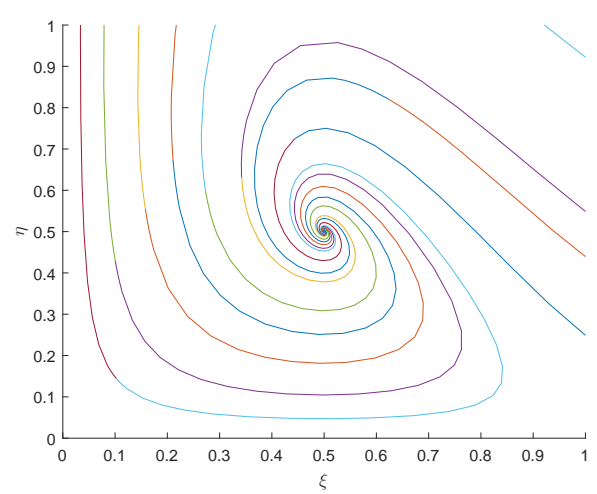

(a)

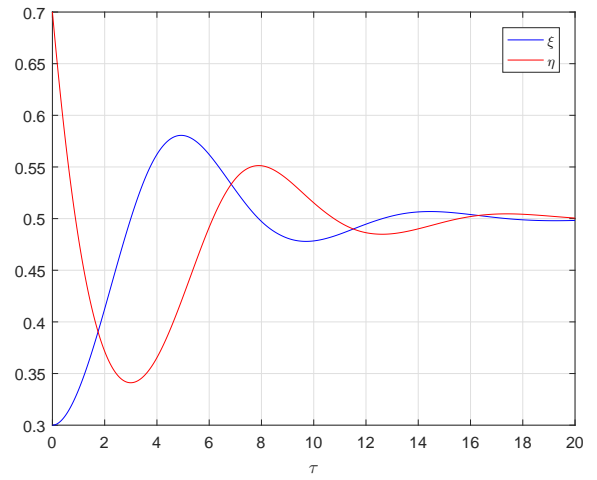

(b)

Figure 1: Wild fishery without aquaculture. (a) The phase portrait of the system (34). Input data: $\gamma_{1}=1, \gamma_{2}=2 . P_{2}=\left(\frac{1}{2}, \frac{1}{2}\right)$ is the stable equilibrium point. (b) The normalized fishery employment, $\eta$ (red curve), and the normalized stock density, $\xi$ (blue curve), as function of the normalized time $\tau$ for the system (34). Input data: $\gamma_{1}=1, \gamma_{2}=2$, and initial condition $\xi(0)=\frac{3}{10}, \eta(0)=\frac{7}{10} . P_{2}=\left(\frac{1}{2}, \frac{1}{2}\right)$ is the stable equilibrium point.

$P_{2}=\left(\frac{\gamma_{1}}{\gamma_{2}}, 1-\frac{\gamma_{1}}{\gamma_{2}}\right)=\left(\frac{1}{2}, \frac{1}{2}\right)$ and represent damped oscillations. This result is in accordance with what one would expect in a wild fishery without aquaculture, where the fishery profit determines the expansion/contraction of the industry effort (employment). From the initial condition, shown in Fig.1b, representing relative high level of effort and relative low stock volume, the fishery will be unprofitable. This will lead to a reduction in effort which is followed by increase in the stock volume, which makes the fishery more profitable causing increased effort, which then leads to reduced biomass density, and so on. Secondly, we study the simplified model with no labor market impact $\left(\gamma_{3}=\gamma_{4}=0\right)$, but now with $\gamma_{5}>0$, i.e. positive relative potential 
net profit in the aquaculture. In this case there is an exponentially increase of $\theta$, and no saturation mechanism in the aquaculture industry. Fig.2 illustrates the fish stock and fishery effort development within the framework of the $2 D$ non - autonomous dynamical system (33). This simulation shows that the fishery development in the initial stage is similar to the development described in Fig.1b. But instead of stabilizing at a sustainable level, the activity will gradually be squeezed by the negative external stock impact following from unbounded aquaculture production development. The final outcome is that the fishery, after a transient phase, will gradually decline, and eventually wound up. The fishing activity (employment) will be unprofitable and close down in finite time, and later on the population (fish stock) will go extinct. Notice that this result, is based on the special case assuming unbounded growth in aquaculture effort, following from permanent positive relative net profit in the aquaculture $\left(\gamma_{5}>0\right)$. The saturation mechanism via effort market $\mu>0$ competition alter will prevent such a development. Next, we consider the system (36) - (37) and the $\varepsilon=0$ - limit of the same system. As pointed out, these simplified models describe the special case with no or weakly impact from aquaculture on wild fish biomass growth. Compared to the simplified versions detailed earlier in this subsection, the labor market mechanism in this modeling approach involves a saturating impact on the effort in both industries. In our numerical study we make use of the parameters in Set 1 except that $\gamma_{4}$ - value is replaced by $\varepsilon=0.1$. The corresponding interior equilibrium point $\tilde{\mathbf{x}}_{e}$ of the unperturbed system (36) - 


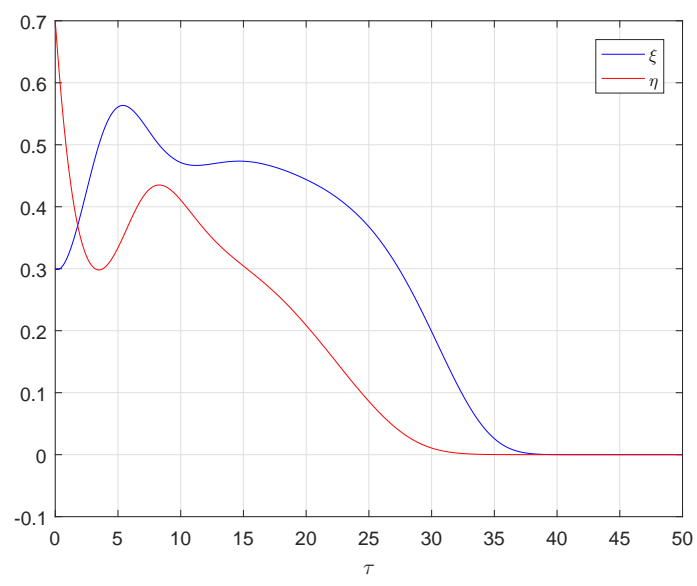

Figure 2: Wild fishery without labour market competition $(\mu=0)$ and steady growing aquaculture $\left(\gamma_{5}>0\right)$. The normalized fishery employment, $\eta$ (red curve), and the normalized stock density, $\xi$ (blue curve) for the $2 D$ non-autonomous system (33) as function of the normalized time $\tau$ in case of permanent positive aquaculture growth. Input data: $\gamma_{1}=1, \gamma_{2}=2, \gamma_{5}=\frac{1}{10}$ and initial condition $\xi(0)=\frac{3}{10}, \eta(0)=\frac{7}{10}, \theta(0)=\frac{5}{100}$.

(37) is computed by means of the expression for $P_{4}$ listed in Table 5 . We get

$$
\tilde{\mathbf{x}}_{e}=\left(\tilde{\xi}_{e}, \tilde{\eta}_{e}, \tilde{\theta}_{e}\right)=\left(\frac{11}{20}, \frac{9}{20}, \frac{13}{400}\right)
$$

Fig. 3 compares the dynamical evolution of the system (36) - (37) with the outcome of the $\varepsilon=0$ - limit of the same system. The evolution depicted in Fig.3 is approaching the equilibrium point (41) in agreement with the stability theory worked out in Subsection 3.4.2. Fig.3 also illustrates an example with relative high initial employment levels in both industries. The effort market competition will increase the unit cost of effort and will cause a reduced profitability in both industries. This will cause a reduction and stabilisation at a relative low level for aquaculture employment. The effort 


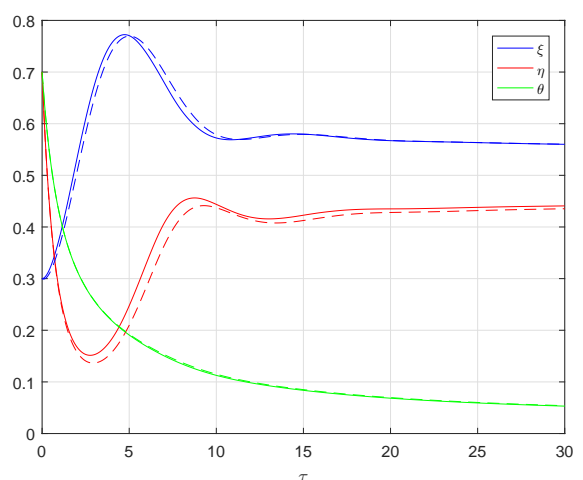

Figure 3: Low effect on biomass growth $(\alpha \rightarrow 0)$. The normalized stock density, $\xi$ (blue curve), the normalized fishery employment, $\eta$ (blue curve), and the normalized fishery employment, $\theta$ (green curve), as function of the normalized time $\tau$, for the system (36) (37) with $\varepsilon=0$ (Bold curve) and the system (36) - (37) with $\varepsilon=\frac{1}{10}$ (Dotted curve). Input data: $\gamma_{1}=1, \gamma_{2}=2, \gamma_{3}=\frac{15}{100}, \gamma_{5}=\frac{1}{10}, \gamma_{6}=1$. Initial condition $\xi(0)=\frac{3}{10}, \eta(0)=\frac{7}{10}$, $\theta(0)=\frac{7}{10}$. The integral curves approach the stable equilibrium point (41).

and stock density for the fishery also stabilize after a transient stage consisting of damped oscillations similar to the mechanism described in Fig.1b. We also notice that the effort level in fishery stabilizes at a lower level compared to Fig.1b because of the cost increase. This lower effort level will result in stabilization of the stock volume at a higher level compared to Fig.1b.

Fig.3 shows that the integral curves of the system (36) - (37) appear as slight continuous deformation of the corresponding curves in the $\varepsilon=0$ limit of (36) - (37), in accordance with the structural stability property of the system (36) - (37).

Finally we do numerical runs of the full model (13) - (16). We make use of the two parameter sets given in Table 7 . Set 1 and Set 2 in Table 7 yield 


\begin{tabular}{ccccccc}
\hline \hline Parameters & $\gamma_{1}$ & $\gamma_{2}$ & $\gamma_{3}$ & $\gamma_{4}$ & $\gamma_{5}$ & $\gamma_{6}$ \\
\hline \hline Set 1 (Stable case) & 1 & 2 & $\frac{15}{100}$ & $\frac{3}{10}$ & $\frac{1}{10}$ & 1 \\
\hline Set 2 (Unstable case) & 1 & 2 & $\frac{3}{10}$ & $\frac{15}{100}$ & $\frac{1}{10}$ & 1 \\
\hline
\end{tabular}

Table 7: Parameter sets underlying the numerical simulations of the model (13).

the interior equilibrium points

$$
\left(\xi_{e}, \eta_{e}, \theta_{e}\right)=\left(\frac{11}{20}, \frac{7}{30}, \frac{13}{60}\right)
$$

and

$$
\left(\xi_{e}, \eta_{e}, \theta_{e}\right)=\left(\frac{11}{20}, \frac{13}{60}, \frac{7}{30}\right)
$$

respectively. The equilibrium point (42) is asymptotically stable, whereas the equilibrium point (43) is unstable, The simulations confirm the predictions deduced from the stability analysis. In Fig.4a and Fig.4b the evolution of the density volume is depicted in the stable case $\left(\gamma_{3}<\gamma_{4}\right)$ as bold curves, and the unstable case $\left(\gamma_{3}>\gamma_{4}\right)$ as dotted curves. For Set 1 in Table 7 , the integral curves approach the equilibrium point (42), independently of the initial conditions.

For the unstable case with Set 2 in Table 7 the dynamical evolution is somewhat more complicated. The numerical simulations in this case indicate that the two boundary equilibrium points $Q_{3}$ and $Q_{4}$ introduced in Subsection 3.2 play a fundamental role in this case. For Set 2 these two points are given as

$$
Q_{3}=\left(\xi_{e}, \eta_{e}, \theta_{e}\right)=\left(\frac{1}{3}, 0, \frac{2}{3}\right)
$$


and

$$
Q_{4}=\left(\xi_{e}, \eta_{e}, \theta_{e}\right)=\left(\frac{13}{23}, \frac{10}{23}, 0\right)
$$

Moreover, our previous findings show that these two equilibrium points are stable for Set 2. A notable feature is that in this case we get integral curves which will either settle down on $Q_{3}$ or on $Q_{4}$, depending on the initial conditions: In Fig.4a where we have chosen a relative low initial level of employment in both industries i.e. $\eta(0)<\eta_{e}$ and $\theta(0)<\theta_{e}$, we get a decreasing aquaculture employment which eventually wound up. In this case the corresponding solution ends up on $Q_{4}$. In Fig.4b we have selected a somewhat relative higher initial level of employment in both industries $\eta(0)>\eta_{e}$ and $\theta(0)>\theta_{e}$. Here the unstable parameter regime gives rise to an increase in the aquaculture production to a substantial higher level, and the fishery employment will gradually decline and eventually be wounded up, and the stock volume stabilizes on a substantial lower level. In this case the integral curves end up on $Q_{3}$. We have carried out more numerical simulations in the regime $\gamma_{3}>\gamma_{4}$ and where $Q_{3}$ and $Q_{4}$ are asymptotically stable boundary equilibrium points. These simulations support the idea that there always is a proper subset $\Omega_{+}$of $\Sigma_{+}$which acts as an attraction basin for $Q_{3}$ whereas the absolute complement $\Omega_{+}^{\mathcal{C}}=\Sigma_{+} \backslash \Omega_{+}$of this subset in $\Sigma_{+}$will be an attraction basin for $Q_{4}$. We do not pursue any further analysis of this problem here, however. 


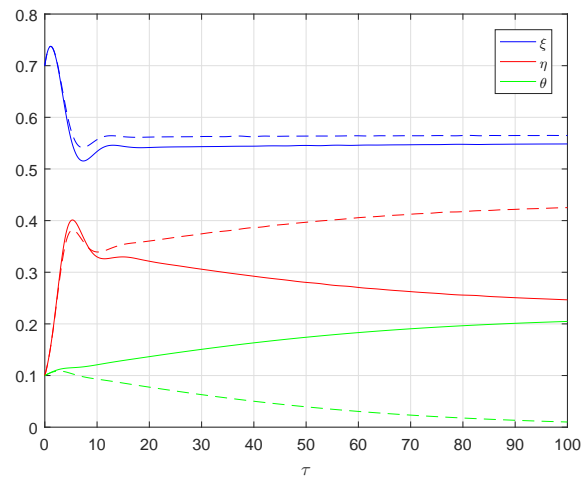

(a)

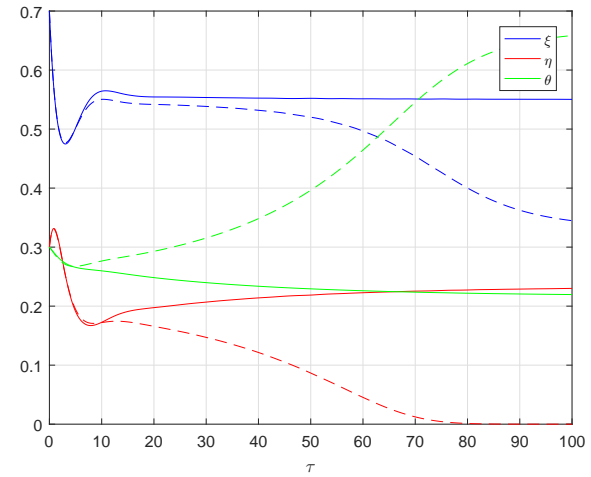

(b)

Figure 4: Stable and unstable cases. (a) The normalized stock density, $\xi$ (blue curve), the normalized fishery employment, $\eta$ (red curve), and the normalized aquaculture employment, $\theta$ (green curve) for the system (13) as function of the normalized time $\tau$ for Set 1 (bold curves) and Set 2 (dashed curves) in Table 7. Set 1 and Set 2 produce the interior equilibrium points (42) and (43), respectively. Initial conditions: $\xi(0)=\frac{7}{10}, \eta(0)=\frac{1}{10}$, $\theta(0)=\frac{1}{10}$. The integral curves approach $Q_{4}$ given by (45) for Set 2. (b) The same integral curves for the system (13) as in 4a. New initial conditions: $\xi(0)=\frac{7}{10}, \eta(0)=\frac{3}{10}$, $\theta(0)=\frac{3}{10}$. The integral curves approach $Q_{3}$ given by (44) for Set 2 .

\subsection{Expansive sea farming policy: Increasing the areas for aquaculture pro-} duction

It is possible to study the effect on different equilibrium states by changing the public regulation facing the two sea food industries, within the present modeling framework. As mentioned in the introduction, the area accessible for sea farming is normally controlled by the public authorities. Hence, an example of an expansive policy for increasing the sea farming production, could be that these authorities decide to increase the area available for aqua- 


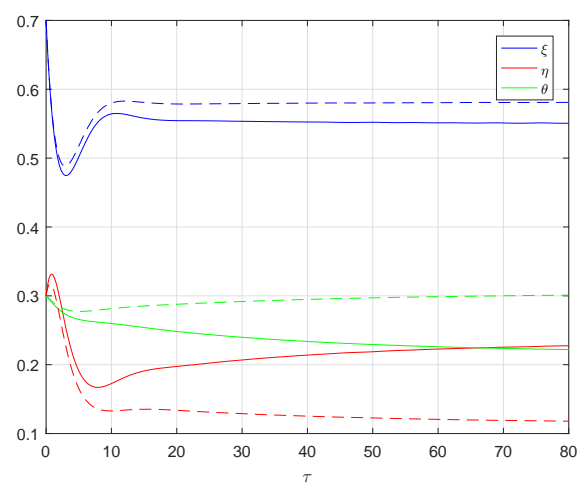

Figure 5: Impact of aquaculture area. The normalized stock density, $\xi$ (blue curve), the normalized fishery employment, $\eta$ (red curve), and the normalized aquaculture employment, $\theta$ (green curve) for the system (13) - (16) as function of the normalized time $\tau$. Input data: Set 1 in Table 7 (bold curve) as reference and the impact of 5 percent increased area $a$ yields $\gamma_{1}=1 \gamma_{2}=1.9 \gamma_{3}=0.158 \gamma_{4}=0.286 \gamma_{5}=0.105 \gamma_{6}=1$. (dashed curve). Initial conditions: $\xi(0)=\frac{7}{10}, \eta(0)=\frac{3}{10}, \theta(0)=\frac{3}{10}$. The equilibrium value for Set 1 is given by (42). Equilibrium point with an increase of $a$ with 5 percent: $\xi_{e} \approx 0.582, \eta_{e} \approx 0.114$, $\left.\theta_{e} \approx 0.304\right)$

culture industry. We proceed as follows: From the definitions in (12) we notice that only $\gamma_{2}, \gamma_{3}, \gamma_{4}$ and $\gamma_{5}$ depend on the area $a$. Let us further assume a policy change, interpreted as a 5 percent increase in aquaculture area a. From the definition of $\gamma_{2}, \gamma_{3}, \gamma_{4}$ and $\gamma_{5}$, we compute the new values of these four nondimensional parameters. Fig. 5 demonstrates the outcome of this change: The bold curves are the integral curves of the system (13) - (16) when using Set 1 in Table 7 as input parameters whereas the dashed curves are the integral curves of the system after a 5 percent increase in the aquaculture area $a$. It is easily seen that the position of the stable equilibrium 
is changed, implying a higher aquaculture employment, a reduced fishery employment, and normalized stock densities on slightly higher levels than before. The explanation is that an increase in the aquaculture area, leads to two direct effects on the industrial efficiencies in harvesting and aquaculture, both reducing the relative profitability in the fishery. First, it is seen from the production function in (4) that the sea farming efficiency increases, and, from (3) the resources in harvesting become more inefficient. The values chosen in our example, however, exhibit a new stable equilibrium where sea farming has increased, the fisheries have decreased and the fish stock stabilizes on a slightly higher level than before. As discussed the reasoning connected to our stable equilibrium in example in Fig. 5 is dependent on a case where $\frac{\gamma_{3}}{\gamma_{4}}<1$, meaning $a<a_{*}$. In a situation where the increase in the accessible area for sea farming is so high that we after implementing the expansive policy now get $a>a_{*}\left(\Leftrightarrow \frac{\gamma_{3}}{\gamma_{4}}>1\right)$, the stable equilibrium changes to an unstable equilibrium. If this is the case we may end up in a stable equilibrium on the boundary $\left(Q_{3}\right)$ where the aquaculture industry is the only sea food industry surviving like the one we illustrated in Fig.4b.

\section{Concluding remarks and extensions}

In the conceptual dynamical model (9) - (11), we have focused on three types of possible interactions within the seafood industries. First of all, we suppose that a production increase in the aquaculture industry is likely to reduce the growth rate in the exploited wild fish stock. Secondly, by increasing the area available for sea farming, one could reduce the productivity 
in harvesting. In addition to these two negative externalities between the sectors, we assume that there might be a competition between these two marine industries for input resources. Such an input market interaction means that the price for input increases (decreases) when one of the industries is increasing (decreasing) the input demand, which then may reduce (increase) the profit of producing the other seafood. Based on the model (9) - (11), we have identified biological and economic structural conditions for which different equilibrium states exist. One possibility is that both the traditional fishery and the aquaculture industry are profitable in the long run and will coexist in the future. A crucial condition for such a stable equilibrium to exist, is that the relative growth impact on fishery from sea farming production (in our model measured by means of the ratio $\frac{\gamma_{3}}{\gamma_{4}}$ ) is not too high (in our model less than 1). Another way of expressing this is that the aquaculture production impact (per unit of effort) on wild fish population growth (measured by means of $\sigma \alpha r a$ ) must be below the potential productivity (per unit of effort) in the fishing industry (measured by means of $q K=(\rho-\varrho a) K)$.

Unlike many other papers in fishery dynamics, we have also discussed unstable equilibrium states. An unstable equilibrium is identified when the aforementioned condition regarding this relative growth impact is not satisfied, i.e. cases where the sea farming has a relatively high impact on the growth potential of the fishery. Dependent on the initial values of the industries effort and the fish stock, we then may end up in stable equilibrium states where one of the industries is steadily decreasing and eventually disappear in the long run. In a numerical example, we have seen that when the effort val- 
ues are equal and initially relatively low, we end up in a stable equilibrium where the aquaculture industry is wiped out. The explanation for this is simply that a relatively low level of the effort in the fishery and aquaculture industry initially, makes harvesting more profitable. This increases fisheries ability to compete for labor. However, when both effort levels are higher, the opposite may happen, and in the long run there is no fishing activity. The explanation of this outcome is that a relatively high level of the effort in both industries cause negative externalities and effort costs which eventually makes harvest activity unprofitable.

We have also seen that allocating more area to aquaculture (in order to develop the sea farming industry) implies a lower activity in the fishery. Larger area gives directly increased productivity in sea farming. The following profitability will increase the effort and the production in the aquaculture, and may lead to three inhibitory effects on the fishery: Reduction in the biomass growth, lower harvest efficiency and tougher effort market competition. Furthermore, we have also argued that if the expansion of sea farming area becomes too high, we may have a situation where the aquaculture activity leads to steadily reduction of the fishery. Eventually, both harvest activity and fish stock could be wiped out in a long run.

Notice that we have not investigated the bistability issue from a rigorous, mathematical perspective i.e. the coexistence of two stable equilibrium states in $\Sigma_{+}$and on its boundary. This problem has been investigated in a multitude of Lotka - Volterra models and their extensions. See for example Smith (1995), Hofbauer and Sigmund (1998) and Kozlov et al. (2016). In 
a future work we aim at studying the convergence of the solutions toward $\mathbf{x}_{e}$ or the equilibrium states on the boundary as well as the possibility of having periodic or chaotic attractors. This problem is indeed related to the decomposition of $\Sigma_{+}$into attraction basins for such attractors.

We will emphasize that our reasoning is based on a simplified and conceptual model where we have made other simplifying assumptions. For instance, we only consider one typical exploited wild fish stock and one sea farming activity. In reality, coastal areas and oceans consist of rather complex ecological and physical systems that need to be understood and modelled properly. For instance, an interesting extension of our model would be to include fodder supply to aquaculture from feed fish where an edible fish feeds on the same stock as the fodder supply depends on. See for instance Hannesson (2003) and Regnier and Schubert (2016) for such modeling reasoning. Another possible extension could be to account for a complete description of the resource level based on the chemostat condition. Here one could make use of the modelling approach for foodwebs suggested by Drossel et al. (2004) and Martinez et al. (2006). We conjecture that this will alter the equilibrium and stability results detected in the present paper.

Moreover, in addition to the two specified marine industries, many other economic activities are located in the coastal zones that also may interact with both fisheries and aquaculture, and thereby affecting the dynamic structure and possible outcomes. For instance, some activities may cause marine emissions that harm the fish growth, where also the history of emissions could be of importance. Marine emissions could be taken into account by modify- 
ing our model by including their temporal influence in the logistic part of the model i.e. in (9). This may change the dynamical features of the model. See for instance Cushing (2013) and the references therein for further details.

In addition to ecological interdependence between fisheries and aquaculture, there are many studies modelling economic market interactions through price mechanisms in the product markets, see e.g. the model proposed by Regnier and Schubert (2016). They show that when biological interactions are moderate the aquaculture is beneficial in the long run. In their model it improves consumer utility and alleviates the pressure on the commercial fish stock. In addition to assume a competition in the input market, we could also have modelled the sea food products stemming from fishery and aquaculture as perfect substitutes in consumption, and proposed a linear sloped demand curve for sea food. The competition following from such an output market would be analogous to the input market competition in the sense that it brings economical dynamics between the two industries. Such a adjusted model will have some of the same market interaction characteristics as our model where the sectors compete for resources in the input market. The similarity means that both input competition and possibly also the competition in the consumption market, means that growth in one of the industries, giving an increase in the input price or a reduction in the output price, reduces the profitability in the other industry.

As in other studies, the present analysis is carried out under the presumption that the industries are expanding (contracting) when profits are positive (negative). In reality, both fisheries and aquaculture are often regu- 
lated by quotas and licenses, normally resulting in at least temporary quota and license profits within the industries. In our model, this means that $\lambda_{x}$ and $\lambda_{s}$ are quite low, or that the regulatory policies have direct effects on the adjustment speed parameters $\left(\lambda_{x}\right.$ and $\left.\lambda_{s}\right)$. However, in future investigations one should look at social preferable allocations of possible stable equilibrium states between the traditional fishery and the sea farming industry. Public regulatory mechanisms in such modeling attempts could be, in addition to decide on available areas for aquaculture production, taxes and subsidies on harvest and/or seafood production in farming.

Developing the model further, taking into account one or more of the possibly complicating aspects mentioned above, might be seen as an interesting task for future research on fishery - aquaculture dynamics.

\section{Bibliography}

Abate, T. G., Nielsen, R., and Tveterås, R. (2016). Stringency of environmental regulation and aquaculture growth: A cross-country analysis. Aquaculture Economics $\& 5$ Management, 20(2):201-221.

Anderson, J. L. (1985). Market interactions between aquaculture and the common-property commercial fishery. Marine Resource Economics, $2(1): 1-24$.

Burridge, L., Weis, J. S., Cabello, F., Pizarro, J., and Bostick, K. (2010). Chemical use in salmon aquaculture: a review of current practices and possible environmental effects. Aquaculture, 306(1-4):7-23.

Chakraborty, K., Jana, S., and Kar, T. (2012). Effort dynamics of a delay-induced prey-predator system with reserve. Nonlinear Dynamics, 70(3):1805-1829.

Clark, C. (2010). Mathematical bioeconomics. The Mathematics of Conservation. New York, NY (USA); John Wiley and Sons Inc. 
Cushing, J. M. (2013). Integrodifferential equations and delay models in population dynamics, volume 20. Springer Science \& Business Media.

de Roos, A. M. (2014). Modeling Population Dynamics. University of Amsterdam, The Netherlands. Lecture notes.

Dempster, T., Uglem, I., Sanchez-Jerez, P., Fernandez-Jover, D., BayleSempere, J., Nilsen, R., and Bjørn, P. A. (2009). Coastal salmon farms attract large and persistent aggregations of wild fish: an ecosystem effect. Marine Ecology Progress Series, 385:1-14.

Drossel, B., McKane, A. J., and Quince, C. (2004). The impact of nonlinear functional responses on the long-term evolution of food web structure. Journal of Theoretical Biology, 229(4):539-548.

EU (2012). Innovating for sustainable growth. a bioeconomy for europe. Technical report, European Commision, Directorate-General for Research and Innovation(EU).

FAO (2016). The state of the World Fisheries and Aquaculture 2016. Contributing to food security and nutrition for all. Food and Agriculture Organization of the United Nations (FAO), Rome.

Flaaten, O. (2010). Fisheries economics and management. Norwegian College of Fishery Science.

Flaaten, O. (2018). Fisheries economics and management. Ola Flaaten and bookboon.com, second edition.

Foley, N. S., Armstrong, C. W., Kahui, V., Mikkelsen, E., and Reithe, S. (2012). A review of bioeconomic modelling of habitat-fisheries interactions. International Journal of Ecology, 2012:1-11.

Ghosh, B. and Kar, T. (2014). Sustainable use of prey species in a preypredator system: Jointly determined ecological thresholds and economic trade-offs. Ecological Modelling, 272:49-58.

Guckenheimer, J. and Holmes, P. (1983). Nonlinear oscillations, dynamical systems, and bifurcations of vector fields. Springer-Verlag.

Hannesson, R. (1983). Optimal harvesting of ecologically interdependent fish species. Journal of Environmental Economics and Management, 10(4):329-345. 
Hannesson, R. (2003). Aquaculture and fisheries. Marine policy, 27(2):169 178.

Hersoug, B. (2012). The fight for space on the coast - a historical sketch(kampen om plass p kysten - en historisk skisse). In Hersoug, B. and Johnsen, J. P., editors, The fight for space on the coast(Kampen om plass p kysten. Interesser og utviklingstrekk $i$ kystsoneplanleggingen). Universitetsforlaget. (In Norwegian).

Hoagland, P., Jin, D., and Kite-Powell, H. (2003). The optimal allocation of ocean space: aquaculture and wild-harvest fisheries. Marine Resource Economics, 18(2):129-147.

Hofbauer, J. and Sigmund, K. (1998). Evolutionary games and population dynamics. Cambridge university press.

Hurwitz, A. (1964). On the conditions under which an equation has only roots with negative real parts. Selected papers on mathematical trends in control theory, 65:273-284.

Jiang, S. (2010). Aquaculture, capture fisheries, and wild fish stocks. Resource and Energy Economics, 32(1):65-77.

Kozlov, V., Vakulenko, S., and Wennergren, U. (2016). Stability of ecosystems under invasions. Bulletin of mathematical biology, 78(11):2186-2211.

Liu, Y., Olaussen, J. O., and Skonhoft, A. (2014). Fishy fish? the economic impacts of escaped farmed fish. Aquaculture Economics \&s Management, 18(3):273-302.

Logan, J. D. (1987). Applied Mathematics: A Contemporary Approach, New York: J. New York, NY (USA); John Wiley and Sons Inc.

Lorenzen, K., Beveridge, M., and Mangel, M. (2012). Cultured fish: integrative biology and management of domestication and interactions with wild fish. Biological Reviews, 87(3):639-660.

Martinez, N. D., Williams, R. J., and Dunne, J. A. (2006). Diversity, complexity, and persistence in large model ecosystems. Ecological networks: linking structure to dynamics in food webs, pages 163-185. 
McCausland, W., Mente, E., Pierce, G., and Theodossiou, I. (2006). A simulation model of sustainability of coastal communities: aquaculture, fishing, environment and labour markets. Ecological Modelling, 193(3):271294.

Mikkelsen, E. (2007). Aquaculture-fisheries interactions. Marine Resource Economics, 22(3):287-303.

Murray, J. D. (2002). Mathematical biology I: An Introduction, Third Edition, Vol. 17 Interdisciplinary Applied Mathematics. Springer, New York, NY, USA,.

NFD (2015). Forutsigbarhet og miljømessig bœrekraftig vekst i norsk lakseof $\varnothing$ rretoppdrett. White paper 16 (2014-2015), Ministry of Trade, Industry and Fisheries (NFD). (in Norwegian).

Osmundsen, T. C., Almklov, P., and Tveterås, R. (2017). Fish farmers and regulators coping with the wickedness of aquaculture. Aquaculture Economics 85 Management, 21(1):163-183.

Perrings, C. (2016). The economics of the marine environment: A review. Environmental Economics and Policy Studies, 18(3):277-301.

Read, P. and Fernandes, T. (2003). Management of environmental impacts of marine aquaculture in europe. Aquaculture, 226(1):139-163.

Regnier, E. and Schubert, K. (2016). To what extent is aquaculture socially beneficial? a theoretical analysis. American Journal of Agricultural Economics, 99(1):186-206.

Smith, H. (1995). Monotone dynamical systems: An introduction to competitive and cooperative systems, ams math. surveys and monographs, vol. 41. American Mathematical Society, Providence RI.

Smith, V. L. (1969). On models of commercial fishing. Journal of political economy, $77(2): 181-198$.

Steinset, T. A. (2017). From supplementary industry to billion industry (frå attåtnæring til milliardindustri). Samfunnsspeilet, SSB, (1/2017). (In Norwegian). 
Steinshamn, S. I. (2017). Predators in the market: implications of market interaction on optimal resource management. Journal of Bioeconomics, 19(3):327-341.

Svåsand, T., Kvamme, B. O., Stien, L. H., Taranger, G. L., and Boxaspen, K. K. (2016). Risk assessment of Norwegian fish farming. 2016 (Risikovurdering av norsk fiskeoppdrett. 2016. Institute of Marine Research. (In Norwegian).

Vasil'eva, A. B., Butuzov, V. F., and Kalachev, L. V. (1995). The boundary function method for singular perturbation problems, volume 14. Siam.

Whitmarsh, D. J., Cook, E. J., and Black, K. D. (2006). Searching for sustainability in aquaculture: an investigation into the economic prospects for an integrated salmon-mussel production system. Marine Policy, 30(3):293-298.

Ye, Y. and Beddington, J. R. (1996). Bioeconomic interactions between the capture fishery and aquaculture. Marine Resource Economics, 11(2):105123.

\section{Acknowledgements}

The authors are grateful to Professor Ola Flåten (The Arctic University of Norway) and Professor Arcady Ponosov (Norwegian University of Life Sciences) for fruitful and stimulating discussions during the preparation phase of this paper. The authors would also like to thank the anonymous reviewers on the first version of this paper for constructive remarks. This research was supported by The Arctic University of Norway, Norwegian University of Life Sciences, and The Research Council of Norway, project number 239070. 


\section{Appendix A. Equilibrium and stability analysis in the transition case $\gamma_{3}=\gamma_{4}$.}

Notice that our system possesses the equilibrium points $Q_{0}, Q_{1}, Q_{2}, Q_{3}$ and $Q_{4}$ given by $(21)$ even in the transition case $\gamma_{3}=\gamma_{4}$ (corresponding to $\operatorname{det}(\mathbf{A})=0)$. If

$$
\gamma_{3}=\gamma_{4}, \quad \frac{\gamma_{2}-\gamma_{1}}{\gamma_{2}+\gamma_{3}} \neq \frac{\gamma_{5}}{\gamma_{3} \gamma_{6}}
$$

we have no solutions of the linear system of equations (24). Hence we have no equilibrium point inside the first octant $\Sigma_{+}$in this case. For the complementary situation

$$
\gamma_{3}=\gamma_{4}, \quad \frac{\gamma_{2}-\gamma_{1}}{\gamma_{2}+\gamma_{3}}=\frac{\gamma_{5}}{\gamma_{3} \gamma_{6}}
$$

the system (24) has infinitely many solutions. We find that these solutions are located on the line segment

$$
\xi_{e}=\frac{\gamma_{1}+\gamma_{3}}{\gamma_{2}+\gamma_{3}}, \quad \eta_{e}+\theta_{e}=\frac{\gamma_{2}-\gamma_{1}}{\gamma_{2}+\gamma_{3}}, \quad \gamma_{2} \geq \gamma_{1}>0
$$

Here either $\eta_{e}$ or $\theta_{e}$ are free parameters satisfying the restrictions

$$
0<\eta_{e}, \theta_{e}<\frac{\gamma_{2}-\gamma_{1}}{\gamma_{2}+\gamma_{3}}
$$

Notice that $Q_{3}$ and $Q_{4}$ are located on this line segment. The boundary point $Q_{2}$ with no fishery and no aquaculture effort, belongs to this line segment if $\gamma_{1}=\gamma_{2}\left(\Leftrightarrow \gamma_{5}=0\right)$, which is reasonable since this condition corresponds to the zero profit case for both industries.

In the degenerate case $\gamma_{3}=\gamma_{4}$, we first notice that in the case with nonexistence of equilibrium points inside $\Sigma_{+}$(i.e. when the condition (A.1) 
is fulfilled) the stability results remain the same as in the case $\gamma_{3} \neq \gamma_{4}$. The degenerate case with the extra constraint (A.2) yields the following results: The Jacobian evaluated the equilibrium point (A.3) is zero, which means that at least one of the eigenvalues is zero, while the remaining two eigenvalues are negative or have negative real part. Hence the stability test based on linearization is inconclusive in this case. For the boundary points $Q_{0}, Q_{1}$ and $Q_{2}$ we get the same results as summarized in Table 3, whereas for the equilibrium points $Q_{3}$ and $Q_{4}$ it is not possible to assess the stability by means of the linearization since both these two points are located on the line segment (A.3).

\section{Appendix B. Stability analysis of the equilibrium point on the boundary and in the interior of $\Sigma_{+}$when $\gamma_{3} \neq \gamma_{4}$.}

We examine the stability properties of the equilibrium points $Q_{0}, Q_{1}$, $Q_{2}, Q_{3}$ and $Q_{4}$ belonging to the boundary of the first octant $\Sigma_{+}$, i.e. the points given by (21) when $\gamma_{3} \neq \gamma_{4}$. In order to do that we must compute the Jacobian of the vectorfield (17) evaluated at each of these five equilibrium points and determine the location of the eigenvalues in each of these five cases. Table 3 which summarizes the outcome of the stability analysis, is based on the properties of these eigenvalues. We have taken into account the fact that linear stability analysis is applicable to assess the stability of these equilibrium points provided the real part of all the eigenvalues of the actual Jacobian is nonzero, in accordance with Hartman - Grobmans theorem (Guckenheimer and Holmes, 1983). An additional notable feature 
is the existence of parameter regimes which represent the transition between stability and instability of the corresponding equilibrium point. Here at least one of the eigenvalues of the Jacobian evaluated the equilibrium point is zero, which means that the actual equilibrium point is subject to a codimension - 1 bifurcation. The stability predictions cannot be based on the linearized dynamics in this case. One has to take nonlinear terms into account when dealing with the stability problem. We do not pursue this problem here, however.

Here we prove the fact that the equilibrium point $\mathbf{x}_{e}$ given by (25) is asymptotically stable (unstable) if $\gamma_{4}>\gamma_{3}\left(\gamma_{4}<\gamma_{3}\right)$. It is tacitly assumed that we are in a parameter regime for the $\gamma_{i}, i=1,2, . ., 6$ for which are guaranteed that $\mathbf{x}_{\mathbf{e}} \in \boldsymbol{\Sigma}_{+}$. We proceed as follows: The Jacobian $\mathbf{J}_{e}$ of the vector field $\mathbf{F}$ of the dynamical system (17) - (18) evaluated at the equilibrium point $\mathbf{x}_{e}$ is given by

$$
\mathbf{J}_{e}=-\mathbf{D}\left(\mathbf{x}_{e}\right) \mathbf{A}=\left(\begin{array}{ccc}
-\xi_{e} & -\xi_{e} & -\xi_{e} \\
\gamma_{2} \eta_{e} & -\gamma_{3} \eta_{e} & -\gamma_{4} \eta_{e} \\
0 & -\gamma_{3} \gamma_{6} \theta_{e} & -\gamma_{4} \gamma_{6} \theta_{e}
\end{array}\right)
$$

The characteristic polynomial $P_{3}$ of $\mathbf{J}_{e}$ is given by

$$
P_{3}(\lambda)=\operatorname{det}\left\{\lambda \mathbf{I}+\mathbf{D}\left(\mathbf{x}_{e}\right) \mathbf{A}\right\}=\lambda^{3}+a_{1} \lambda^{2}+a_{2} \lambda+a_{3}
$$

Here $\mathbf{I}$ is the unit $3 \times 3$ - matrix, whereas the coefficients $a_{1}, a_{2}$ and $a_{3}$ are given as 


$$
\begin{gathered}
a_{1}=\operatorname{tr}\left\{\mathbf{D}\left(\mathbf{x}_{e}\right) \mathbf{A}\right\}=\xi_{e}+\gamma_{3} \eta_{e}+\gamma_{4} \gamma_{6} \theta_{e} \\
a_{2}=\frac{1}{2}\left\{\left(\operatorname{tr}\left\{\mathbf{D}\left(\mathbf{x}_{e}\right) \mathbf{A}\right\}\right)^{2}-\operatorname{tr}\left\{\left(\mathbf{D}\left(\mathbf{x}_{e}\right) \mathbf{A}\right)^{2}\right\}\right. \\
=\left(\gamma_{2}+\gamma_{3}\right) \xi_{e} \eta_{e}+\gamma_{4} \gamma_{6} \xi_{e} \theta_{e} \\
a_{3}=\operatorname{det}\left\{\mathbf{D}\left(\mathbf{x}_{e}\right) \mathbf{A}\right\}=\gamma_{2} \gamma_{6}\left(\gamma_{4}-\gamma_{3}\right) \xi_{e} \eta_{e} \theta_{e}
\end{gathered}
$$

We observe that $a_{3}>0$ if $\gamma_{4}>\gamma_{3}$. Moreover, we find that the Routh Hurwitz determinants $D_{1} \equiv a_{1}$ and $D_{2} \equiv a_{1} a_{2}-a_{3}$ are strictly positive i.e.

$$
\begin{gathered}
D_{1} \equiv a_{1}>0 \\
D_{2} \equiv a_{1} a_{2}-a_{3}=\left(\gamma_{2}+\gamma_{3}\right)\left(\xi_{e}+\gamma_{3} \eta_{e}\right) \xi_{e} \eta_{e} \\
+\left(\xi_{e}+\gamma_{4} \gamma_{6} \theta_{e}\right) \gamma_{4} \gamma_{6} \xi_{e} \theta_{e}+\left(\gamma_{2}+2 \gamma_{3}\right) \gamma_{3} \gamma_{6} \xi_{e} \eta_{e} \theta_{e}>0
\end{gathered}
$$

Then, according to the Routh - Hurwitz criterion, all the zeros of the characteristic polynomial $P_{3}$ is located in the left $\lambda$ - half plane provided $\gamma_{4}>\gamma_{3}$ (Hurwitz, 1964). Hence we arrive at the following conclusion: The equilibrium point $\boldsymbol{x}_{e} \in \Sigma_{+}$is asymptotically stable if $\gamma_{4}>\gamma_{3}$. Next, let us explore the stability problem in the complementary regime $\gamma_{3}>\gamma_{4}$. Here we have $P_{3}(0)=a_{3}<0$. In that case $P_{3}$ has at least one strictly positive zero, which means that the Jacobian $\mathbf{J}_{e}$ always has a one strictly positive eigenvalue. This means that the corresponding equilibrium point $\mathbf{x}_{e}$ is always unstable when $\gamma_{3}>\gamma_{4}$. Finally, let us examine the possibility of having bifurcations of the equilibrium point $\mathbf{x}_{e}$ in the regime $\gamma_{3} \neq \gamma_{4}$. First we notice that since by $(26)$ we have $\operatorname{det}(\mathbf{A}) \neq 0$ and $\operatorname{det}\left(\mathbf{D}\left(\mathbf{x}_{e}\right)\right)=\xi_{e} \eta_{e} \theta_{e}>0$, the equation (25) shows that $\operatorname{det}\left(\mathbf{J}_{\mathbf{e}}\right) \neq \mathbf{0}$. This means that the $\lambda=0$ is not an eigenvalue of the Jacobian $\mathbf{J}_{\mathbf{e}}$. Hence we have no static bifurcation 
at the equilibrium point $\mathbf{x}_{e}$. The previous arguments show that no Hopf bifurcation will take place the parameter regime $\gamma_{3} \neq \gamma_{4}$, either.

The threshold conditions for stability in Table 3 have the following interpretations: The threshold condition for $Q_{3}$ reads

$$
\frac{p_{s} r a}{p_{x} q K}>1-\frac{\alpha r a}{\mu K}\left(p_{s} r a-w\right)
$$

when restoring to the original dimensional parameters. Here the left hand side fraction is interpreted as the relative aquaculture industry profitability. The nominator $\left(p_{s} r a\right)$ is the revenue per unit effort in the aquaculture industry whereas the denominator $\left(p_{x}(\rho-\varrho a) K\right)$ is the potential revenue per unit effort in the fishery. Hence, the equilibrium state with no fishery effort and with a finite aquaculture activity is stable if the relative aquaculture industry profitability exceeds a certain threshold value: $1-\frac{\alpha r a}{\mu K}\left(p_{s} r a-w\right)$. This value is determined by labour market conditions and the biomass growth impact. The threshold value is increasing in $w$ an $\mu$, and decreasing in $\alpha$. The threshold condition for $Q_{4}$ in Table 3 with original parameters can be written as

$$
\left(p_{s} r a-w\right)<\mu \frac{\sigma}{q}\left(\frac{w+\frac{\mu \sigma}{q}}{p_{x} q K+\frac{\mu \sigma}{q}}\right)
$$

where the expression on the left hand side is the net aquaculture industry profit. Hence, the equilibrium state with no aquaculture activity is stable if the aquaculture industry profit is below a certain threshold value: $\mu \frac{\sigma}{q}\left(\frac{w+\frac{\mu \sigma}{q}}{p_{x} q K+\frac{\mu \sigma}{q}}\right)$. This value is determined by several of the dimensional parameters, including those ones representing the labour market conditions and the potential revenue in the fishery. 


\section{Appendix C. Detailed analysis in the case of low aquaculture pro- duction effect on biomass growth.}

Here we present a detailed analysis of the dynamical system (36) - (37) in the regime $0<\varepsilon \ll 1$ can be viewed as a regularly perturbed system. Hence the whole body of theory for such systems is available for the purpose of determining a uniformly valid asymptotic approximation to the solution of the system. To the leading order, we have

$$
\left(\begin{array}{c}
\tilde{\xi}(\tau, \varepsilon) \\
\tilde{\eta}(\tau, \varepsilon) \\
\tilde{\theta}(\tau, \varepsilon)
\end{array}\right)=\left(\begin{array}{c}
\tilde{\xi}_{0}(\tau) \\
\tilde{\eta}_{0}(\tau) \\
\tilde{\theta}_{0}(\tau)
\end{array}\right)+O(\varepsilon)
$$

for this approximation. Here $\tilde{\xi}_{0}, \tilde{\eta}_{0}$ and $\tilde{\theta}_{0}$ are the solutions to the system

$$
\tilde{\xi}_{0}^{\prime}=\tilde{\xi}_{0} F_{0}\left(\tilde{\xi}_{0}, \tilde{\eta}_{0}, \tilde{\theta}_{0}\right), \quad \tilde{\eta}_{0}^{\prime}=\tilde{\eta}_{0} G_{0}\left(\tilde{\xi}_{0}, \tilde{\eta}_{0}, \tilde{\theta}_{0}\right), \quad \tilde{\theta}_{0}^{\prime}=\tilde{\theta}_{0} H_{0}\left(\tilde{\xi}_{0}, \tilde{\eta}_{0}, \tilde{\theta}_{0}\right)
$$

where $F_{0}, G_{0}$ and $H_{0}$ are the linear functions

$$
\begin{gathered}
F_{0}\left(\tilde{\xi}_{0}, \tilde{\eta}_{0}, \tilde{\theta}_{0}\right)=1-\tilde{\xi}_{0}-\tilde{\eta}_{0} \\
G_{0}\left(\tilde{\xi}_{0}, \tilde{\eta}_{0}, \tilde{\theta}_{0}\right)=-\gamma_{1}+\gamma_{2} \tilde{\xi}_{0}-\gamma_{3} \tilde{\eta}_{0}-\tilde{\theta}_{0} \\
H_{0}\left(\tilde{\xi}_{0}, \tilde{\eta}_{0}, \tilde{\theta}_{0}\right)=\gamma_{5}-\gamma_{3} \gamma_{6} \tilde{\eta}_{0}-\gamma_{6} \tilde{\theta}_{0}
\end{gathered}
$$

See for example Vasil'eva et al. (1995) for a general exposition on the theory for regularly perturbed dynamical systems.

Introduce the vector fields $\mathbf{G}_{0}$ and $\mathbf{G}_{1}$ defined as 


$$
\begin{gathered}
\mathbf{G}_{0}(\tilde{\mathbf{x}})=\mathbf{D}(\tilde{\mathbf{x}})\left\{\mathbf{b}-\mathbf{A}_{0} \tilde{\mathbf{x}}\right\} \\
\mathbf{G}_{1}(\tilde{\mathbf{x}})=-\mathbf{D}(\tilde{\mathbf{x}}) \mathbf{A}_{1} \tilde{\mathbf{x}}
\end{gathered}
$$

Here $\mathbf{D}$ and $\mathbf{b}$ are defined as in (18), whereas $\mathbf{A}_{0}$ and $\mathbf{A}_{1}$ are given as

$$
\mathbf{A}_{0}=\left(\begin{array}{ccc}
1 & 1 & 0 \\
-\gamma_{2} & \gamma_{3} & 1 \\
0 & \gamma_{3} \gamma_{6} & \gamma_{6}
\end{array}\right), \quad \mathbf{A}_{1}=\left(\begin{array}{ccc}
0 & 0 & 1 \\
0 & 0 & 0 \\
0 & 0 & 0
\end{array}\right)
$$

The dynamical systems (36) - (37) and (C.2) - (C.3) can then be written on the compact vector form

$$
\frac{d}{d \tau} \tilde{\mathbf{x}}=\mathbf{G}_{0}(\tilde{\mathbf{x}})+\varepsilon \mathbf{G}_{1}(\tilde{\mathbf{x}})
$$

and

$$
\frac{d}{d \tau} \tilde{\mathbf{x}}_{0}=\mathbf{G}_{0}\left(\tilde{\mathbf{x}}_{0}\right)
$$

respectively. Let us then detect the equilibrium points of the unperturbed system (C.6) in the part of the phase space for which $\tilde{\xi}, \tilde{\eta}, \tilde{\theta} \geq 0$ and their corresponding stability properties. We conveniently introduce the set

$$
\Sigma_{+}=\left\{(\tilde{\xi}, \tilde{\eta}, \tilde{\theta}) \in \mathbb{R}^{3} ; \quad \tilde{\xi}, \tilde{\eta}, \tilde{\theta}>0\right\}
$$

We first deal with possible equilibrium points located on the boundary of $\Sigma_{+}$. For the sake of convenience these equilibrium points are listed in Table 5 together with their respective stability properties. The stability analysis underlying the conclusions summarized in Table 5 is based on linearization of the dynamical system (C.6) about the actual equilibrium points $M_{1}, M_{2}$ 
and $M_{3}$ and Hartman - Grobmans theorem. Just as in Subsection 3.3 the linearization procedure fails to give the correct stability prediction for parameter regime producing at least one zero eigenvalue of the corresponding Jacobian i.e. in the regime of transition between stability and instability. Next, let us examine the possibility of having an equilibrium point in the interior of $\Sigma_{+}$. The equation for the equilibrium point is

$$
\mathbf{A}_{0} \tilde{\mathbf{x}}_{e}=\mathbf{b}
$$

This system has a unique solution since $\operatorname{det}\left(\mathbf{A}_{0}\right)=\gamma_{2} \gamma_{6}>0$. The components of $\tilde{\mathbf{x}}_{e}$ are given as

$$
\begin{gathered}
\tilde{\eta}_{e}=1-\tilde{\xi}_{e} \\
\tilde{\theta}_{e}=\left\{\frac{\gamma_{5}}{\gamma_{6} \gamma_{3}}-\left(1-\tilde{\xi}_{e}\right)\right\} \gamma_{3}
\end{gathered}
$$

where

$$
\tilde{\xi}_{e}=\frac{\gamma_{1}}{\gamma_{2}}+\frac{\gamma_{5}}{\gamma_{2} \gamma_{6}}
$$

The point $M_{4}$ in Table 5 is given by the coordinates of $\mathbf{x}_{e}$. In order to have $\tilde{\mathbf{x}}_{e} \in \Sigma_{+}$, we must impose the requirement

$$
1-\frac{\gamma_{5}}{\gamma_{6} \gamma_{3}}<\tilde{\xi}_{e}<1, \quad \gamma_{5} \geq 0
$$

Notice that we have no equilibrium point in $\Sigma_{+}$when $\gamma_{5}<0$.

The Jacobian - matrix $\tilde{\mathbf{J}}_{e, 0}$ evaluated at this equilibrium point is given as

$$
\tilde{\mathbf{J}}_{e, 0}=-\mathbf{D}\left(\tilde{\mathbf{x}}_{e}\right) \mathbf{A}_{0}
$$

The characteristic polynomial of $\tilde{\mathbf{J}}_{e, 0}$ is given as 


$$
P_{3}(\lambda)=\operatorname{det}\left\{\lambda \mathbf{I}+\mathbf{D}\left(\tilde{\mathbf{x}}_{e}\right) \mathbf{A}_{0}\right\}=\lambda^{3}+b_{1} \lambda^{2}+b_{2} \lambda+b_{3}
$$

where

$$
\begin{gathered}
b_{1}=\tilde{\xi}_{e}+\tilde{\eta}_{e} \gamma_{3}+\tilde{\theta}_{e} \gamma_{6} \\
b_{2}=\left(\gamma_{2}+\gamma_{3}\right) \tilde{\xi}_{e} \tilde{\eta}_{e}+\gamma_{6} \tilde{\xi}_{e} \tilde{\theta}_{e} \\
b_{3}=\gamma_{2} \gamma_{6} \tilde{\xi}_{e} \tilde{\eta}_{e} \tilde{\theta}_{e}
\end{gathered}
$$

We readily observe that $D_{1} \equiv b_{1}>0$. Simple computation reveals that the Routh - Hurwitz determinant $D_{2} \equiv b_{1} b_{2}-b_{3}$ is also strictly positive. Hence, by the Routh - Hurwitz criterion (Hurwitz, 1964), we conclude that all the zeros $\lambda_{i}, i=1,2,3$ of $P_{3}$ are located in the left $\lambda$ - halfplane, i.e. $\operatorname{Re}\left\{\lambda_{i}\right\}<$ $0, i=1,2,3$. Hence the equilibrium point $\tilde{\mathbf{x}}_{\mathbf{e}} \in \boldsymbol{\Sigma}_{+}$is asymptotically stable.

We next consider the perturbed system (C.5). The Jacobian of the vector field $\mathbf{G} \equiv \mathbf{G}_{\mathbf{0}}+\varepsilon \mathbf{G}_{\mathbf{1}}$ evaluated at the point $(\tilde{\mathbf{x}}, \varepsilon)=\left(\tilde{\mathbf{x}}_{e}, 0\right)$ is equal to $\tilde{\mathbf{J}}_{e, 0}$. Since $\operatorname{det}\left(\tilde{\mathbf{J}}_{e, 0}\right)=b_{3}=\gamma_{2} \gamma_{6} \tilde{\xi}_{e} \tilde{\eta}_{e} \tilde{\theta}_{e}>0$, we conclude that the Jacobian of $\mathbf{G}$ evaluated at $(\tilde{\mathbf{x}}, \varepsilon)=\left(\tilde{\mathbf{x}}_{e}, 0\right)$ is invertible. Then, by the implicit function theorem, the system (C.5) has a unique $\varepsilon$ - dependent equilibrium point which locally about $\varepsilon=0$ is a smooth function of $\varepsilon$. Since the equilibrium point $\tilde{\mathbf{x}}_{e}$ is a asymptotically stable (hyperbolic) equilibrium point of (C.6), the same holds true for the $\varepsilon$ - dependent equilibrium point of (C.5) when $0<\varepsilon \ll 1$. Moreover, Hartman - Grobmans theorem implies that the unperturbed model 
(C.6) is locally structural stable. This means that the phase portrait of the perturbed system (C.5) in the vicinity of its equilibrium point is mapped one - to - one and onto the phase portrait of the unperturbed system (C.6) in the vicinity of $\tilde{\mathbf{x}}_{e}$. See Guckenheimer and Holmes (1983) for details concerning the general exposition of the theory for hyperbolic equilibrium points and structural stability.

Finally, notice that the definition (39) of $\varepsilon$ implies that the regime $0<$ $\varepsilon \ll 1, \gamma_{3}$ finite corresponds to the regime $\gamma_{4} \gg \gamma_{3}$. According to Subsection 3.3 the equilibrium point $\tilde{\mathbf{x}}_{e}$ in $\Sigma_{+}$is asymptotically stable. Hence the stability findings of this subsection are consistent with that result. 